\title{
Glucose limitation activates AMPK coupled SENP1- Sirt3 signalling in mitochondria for T cell memory development
}

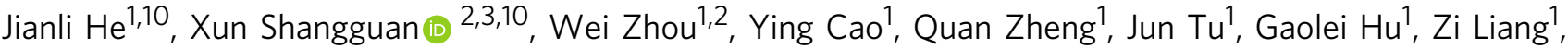 \\ Cen Jiang ${ }^{1}$, Liufu Deng $\mathbb{D}^{3}$, Shengdian Wang ${ }^{4}$, Wen Yang ${ }^{1}$, Yong Zuo ${ }^{1}$, Jiao Ma', Rong Cai ${ }^{1}$, Yalan Chen ${ }^{1}$, \\ Qiuju Fan', Baijun Dong (10 ${ }^{2}$, Wei Xue ${ }^{2}$, Hongsheng Tan ${ }^{5}$, Yitao Qi ${ }^{6}$, Jianmin Gu ${ }^{7}$, Bing Su${ }^{8}$, Y. Eugene Chin ${ }^{9}$, \\ Guoqiang Chen (1) ${ }^{1}$, Qi Wang ${ }^{2 凶}$, Tianshi Wang ${ }^{1 凶}$ \& Jinke Cheng (iD) ${ }^{1 凶}$
}

Metabolic programming and mitochondrial dynamics along with $\mathrm{T}$ cell differentiation affect $\mathrm{T}$ cell fate and memory development; however, how to control metabolic reprogramming and mitochondrial dynamics in T cell memory development is unclear. Here, we provide evidence that the SUMO protease SENP1 promotes T cell memory development via Sirt3 deSUMOylation. SENP1-Sirt3 signalling augments the deacetylase activity of Sirt3, promoting both OXPHOS and mitochondrial fusion. Mechanistically, SENP1 activates Sirt3 deacetylase activity in $T$ cell mitochondria, leading to reduction of the acetylation of mitochondrial metalloprotease YME1L1. Consequently, deacetylation of YME1L1 suppresses its activity on OPA1 cleavage to facilitate mitochondrial fusion, which results in T cell survival and promotes T cell memory development. We also show that the glycolytic intermediate fructose-1,6bisphosphate (FBP) as a negative regulator suppresses AMPK-mediated activation of the SENP1-Sirt3 axis and reduces memory development. Moreover, glucose limitation reduces FBP production and activates AMPK during T cell memory development. These data show that glucose limitation activates AMPK and the subsequent SENP1-Sirt3 signalling for T cell memory development.

\footnotetext{
${ }^{1}$ State Key Laboratory of Oncogenes and Related Genes, Renji Hospital Affiliated; Shanghai Key Laboratory for Tumor Microenvironment and Inflammation, Department of Biochemistry and Molecular Cell Biology, Shanghai Jiao Tong University School of Medicine, Shanghai, China. ${ }^{2}$ Department of Urology, Renji Hospital Affiliated, Shanghai Jiao Tong University School of Medicine, Shanghai, China. ${ }^{3}$ Department of Urology, Xinhua Hospital Affiliated to Shanghai Jiao Tong University School of Medicine, Shanghai, China. ${ }^{4}$ Institute of Biophysics, Chinese Academy of Sciences, Beijing, China. ${ }^{5}$ Clinical Research Center, Shanghai Jiao Tong University School of Medicine, Shanghai, China. ${ }^{6}$ College of Life Sciences, Shaanxi Normal University, Xi'an, Shaanxi, China. ${ }^{7}$ Department of Thoracic Surgery, Zhongshan Hospital, Fudan University, Shanghai, China. ${ }^{8}$ Shanghai Institute of Immunology, Shanghai Jiao Tong University School of Medicine, Shanghai, China. ${ }^{9}$ Institutes of Biology and Medical Sciences, Soochow University Medical College, Suzhou, Jiangsu, China. ${ }^{10}$ These authors contributed equally: Jianli He, Xun Shangguan. ${ }^{\circledR}$ email: wqi@sjtu.edu.cn; tianshi777@shsmu.edu.cn; jkcheng@shsmu.edu.cn
} 
T cells are important contributors to adaptive immunity. Upon stimulation by antigen, $\mathrm{T}$ cells can differentiate into effectors and subsequently form memory cells. In effectors, some subsets, such as memory precursor effector cells (MPECs), differentiate into long-lived memory $\mathrm{T}$ cells and others, such as short-lived effector cells (SLECs), undergo apoptosis after antigen clearance ${ }^{1,2}$. Evidence indicates that the treatment of tumours using long-lived $\mathrm{T}$ cells can improve anti-tumour responses ${ }^{2-4}$, which is similar to results obtained with the use of vaccines against virus infection ${ }^{5}$.

$\mathrm{T}$ cell differentiation status is related to metabolic programming ${ }^{6-8}$. CD8 $8^{+}$effector $\mathrm{T}\left(\mathrm{T}_{\mathrm{E}}\right)$ cells rely on aerobic glycolysis and oxidative phosphorylation (OXPHOS) to support their proliferation ${ }^{9-11}$, whereas memory $\mathrm{T}\left(\mathrm{T}_{\mathrm{M}}\right)$ cells require fatty acid oxidation (FAO) for their survival ${ }^{12-15}$. Nutrient transport and utilization can affect $\mathrm{T}$ cell differentiation fate as well as function ${ }^{16}$. In the metabolic microenvironment, oxygen, nutrients and growth factors are limiting, along with immune reaction of effector T cells ${ }^{16}$. Previous studies have reported that these limiting factors can damage $\mathrm{T}$ cells leading to a gradual loss of their effector function and proliferation capacity ${ }^{17}$. These conditions may trigger $\mathrm{T}$ cells to reprogram their metabolism to ensure proper survival and/or effector function ${ }^{16,18}$.

In addition to metabolic programming, mitochondrial dynamics have been linked to $T$ cell fate ${ }^{7}$. $T_{E}$ cells have punctate mitochondria, whereas $T_{M}$ cells maintain a fused mitochondrial network. As demonstrated using a genetic approach, mitochondrial fusion can facilitate efficient mitochondrial electron transport chain (ETC) activity and $\mathrm{FAO}$ in $\mathrm{T}_{\mathrm{M}}$ cells, but elevates aerobic glycolysis in activated $\mathrm{T}$ cells ${ }^{7,15}$. Moreover, mitochondrial fusion can enhance $T_{M}$ cell survival and activity ${ }^{7}$. Mitochondrial fusion is controlled by mitofusin-1 and mitofusin-2 (Mfn1/Mfn2) in the outer membrane and OPA1 in the inner membrane ${ }^{19-21}$. Interestingly, OPA1-mediated inner membrane fusion but not Mfn1- or Mfn2-mediated fusion is essential for $\mathrm{T}_{\mathrm{M}}$ cell survival ${ }^{7}$. OPAl increases cristae formation to support assembly of the respiratory chain supercomplex and OXPHOS in $\mathrm{T}_{\mathrm{M}}$ cells $^{7}$. However, regulation of OPA1-mediated mitochondrial fusion in T cell memory development is unclear.

AMPK is a critical regulator of $\mathrm{T}$ cell metabolism ${ }^{22}$ and functions as a metabolic checkpoint in effector cells to suppress mTOR activity in nutrient-limited environments ${ }^{23}$. Compared with effector cells, memory $\mathrm{T}$ cells have higher AMPK activity ${ }^{14,23}$. Furthermore, AMPK activation increases FAO and promotes memory development ${ }^{14}$. However, the physiological upstream signals of AMPK activation in T cell memory development are unclear.

Mitochondrial acetylation is a crucial regulatory mechanism for the activation of mitochondrial enzymes ${ }^{24,25}$. Sirt3, as a major mitochondrial $\mathrm{NAD}^{+}$-dependent deacetylase, is required for mitochondrial metabolic adaptation in response to various stresses. Sirt3-knockout mice have markedly increased acetylation levels of mitochondrial proteins in a variety of tissues ${ }^{26,27}$. Sirt3 can target a broad range of mitochondrial proteins ${ }^{26}$ and regulates mitochondrial complex I and complex II to activate electron transport in the mitochondrial inner membrane ${ }^{28}$ and promotes lipid biosynthesis via acetyl-CoA synthase 2 (AceCS2). Sirt3 also enhances FAO via long-chain acyl-CoA dehydrogenase (LCAD) under calorie-restricted conditions ${ }^{29-31}$. Moreover, Sirt3 can trigger global reprogramming of the mitochondrial protein acetylome under calorie-restricted conditions ${ }^{26,31,32}$. In addition to calorie restriction, other cellular stresses modulate Sirt3 function in mitochondrial metabolism ${ }^{32,33}$. In addition, Sirt3 can decrease the reactive oxygen species (ROS) level in mitochondria, indicating that Sirt3 activation is associated with various physiological or pathological processes, such as aging, hearing loss and tumorigenesis ${ }^{34-36}$. However, the function of Sirt3 in T cells is unexplored.

Mitochondrial Sirt3 has been identified as a SUMOylated protein $^{31}$. SUMOylation suppresses Sirt3 deacetylase activity and then affects mitochondrial metabolism ${ }^{31,32}$. In response to calorie restriction, Sentrin-specific protease 1 (SENP1) deSUMOylates Sirt3 in mitochondria ${ }^{31}$ and SENP1-Sirt3 signalling promotes FAO and oxidative phosphorylation in cells and mice ${ }^{31,32}$.This study uncovers that the SENP1-Sirt3 axis is essential for T cell survival and memory development. SENP1-Sirt3 axis promotes FAO-fuelled OXPHOS and reduces YME1L1-mediated OPA1 cleavage in $\mathrm{T}$ cell memory development and T-cell-mediated antitumour immunity. Notably, low glucose activates the SENP1Sirt3 axis via non-classical AMPK signalling in T cells, which promotes $\mathrm{T}$ cell memory development. Importantly, fructose-1,6bisphosphate (FBP) as a glycolytic intermediate suppresses the AMPK-SENP1-Sirt3 signalling in memory development. Therefore, this study reveals that glucose limitation promotes AMPKSENP1-Sirt3 axis via reducing FBP production, leading to enhanced $\mathrm{T}$ cell memory development.

\section{Results}

SENP1-Sirt3 axis is activated during $\mathrm{T}$ cell memory development. Cellular metabolic programme is tightly associated with the mitochondrial protein acetylation ${ }^{29,33,37,38}$. Since effector $\mathrm{T}$ cells $\left(\mathrm{T}_{\mathrm{E}}\right)$ and memory $\mathrm{T}$ cells $\left(\mathrm{T}_{\mathrm{M}}\right)$ perform different metabolic programmes ${ }^{12-15}$, we reasoned that the mitochondrial acetylation pattern in memory $\mathrm{T}$ cells would be different from that in effectors. Indeed, we detected remarkable lower mitochondrial acetylation levels in $\mathrm{CD}^{+} \mathrm{T}_{\mathrm{M}}$ cells than that in $\mathrm{CD}^{+} \mathrm{T}_{\mathrm{E}}$ cells (Fig. 1a and Supplementary Fig. 1a). Sirt3 as a major deacetylase in mitochondria plays a crucial role in regulating mitochondrial protein acetylation, and SUMO-specific protease SENP1 is a crucial regulator of Sirt3 activation via de-SUMOylation ${ }^{31,32}$. We thus speculated that the SENP1-Sirt3 axis may engage in T cell memory development. As expected, we detected the lower level of Sirt3 SUMOylation and more mitochondrial SENP1 proteins in $\mathrm{T}_{M}$ mitochondria than that in $\mathrm{T}_{\mathrm{E}}$ mitochondria (Fig. 1b, c), suggesting that SENP1-Sirt3 signalling is activated along with $\mathrm{T}$ cell memory development. We further confirmed the activation of SENP1-Sirt3 axis by showing the higher mitochondrial acetylation level in $\mathrm{T}_{\mathrm{M}}$ cells with Senp1 conditional knockout (Senp1 cKO) than that in Senp1 wild-type (Senp1 WT) $\mathrm{T}_{\mathrm{M}}$ cells (Fig. 1d and Supplementary Fig. 1b). These data reveal that the SENP1Sirt3 axis is activated in $\mathrm{T}$ cell memory development.

SENP1-Sirt3 axis promotes $\mathrm{T}$ cell memory development. We next assessed whether the SENP1-Sirt3 axis affects T cell memory development. Sirt3 K223R, a SUMOylation mutant, has been reported to mimic the activation of the SENP1-Sirt3 axis $^{31}$. A FACS analysis showed no difference between Sirt3 WT and Sirt3 K223R mice in the thymus $\mathrm{T}$ cell development (Supplementary Fig. 1c, d). However, we found that the central memory $\mathrm{T}$ cells $\left(\mathrm{T}_{\mathrm{CM}}, \mathrm{CD}^{+}{ }^{+} \mathrm{CD}_{4} 4^{+} \mathrm{CD} 2 \mathrm{~L}^{+}\right)$but not naïve $\left(\mathrm{T}_{\mathrm{N}}, \mathrm{CD} 8^{+} \mathrm{CD} 44^{-}\right.$ $\left.\mathrm{CD} 62 \mathrm{~L}^{+}\right)$or effector-memory $\mathrm{T}$ cells $\left(\mathrm{T}_{\mathrm{EM}}, \mathrm{CD} 8^{+} \mathrm{CD} 44^{+}\right.$ CD62 $\mathrm{L}^{-}$) were more abundant in the Sirt3 K223R spleen than that in the Sirt3 WT spleen under unchallenged condition (Fig. 1e, f). Also, the increased memory T cells in Sirt3 K223R spleen under unchallenged condition are 'true' memory $\mathrm{T}$ cells $\left(\mathrm{T}_{\mathrm{M}}, \mathrm{CD} 8^{+}\right.$ $\left.\mathrm{CD} 44^{+} \mathrm{CD} 49 \mathrm{~d}^{+}\right)$but not virtual memory cells $\left(\mathrm{T}_{\mathrm{VM}}\right)\left(\mathrm{CD} 8^{+}\right.$ CD44 ${ }^{+}$CD49d $\left.{ }^{-}\right)^{39,40}$ (Supplementary Fig. 1e, f), and Sirt3 K223R promoted the differentiation of Treg cells in vitro other than $\mathrm{CD} 4^{+}$helper T cells (Supplementary Fig. 1g). We further used Sirt3 K223R-OT1 mouse model to determine the role of SENP1Sirt3 axis in T cell memory development. OT1 $\mathrm{CD} 8^{+}$naïve spleen 
$\mathbf{a}$

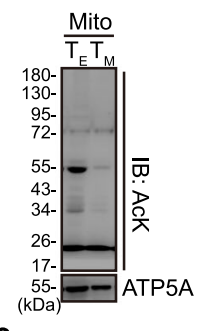

b

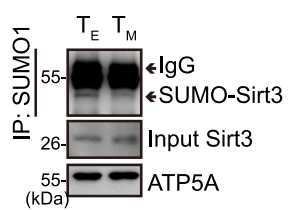

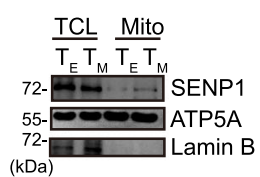

d

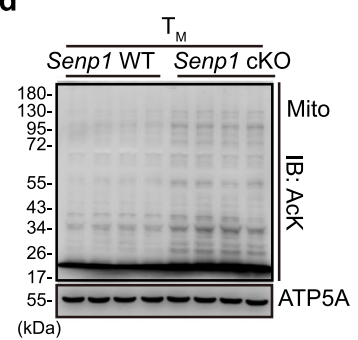

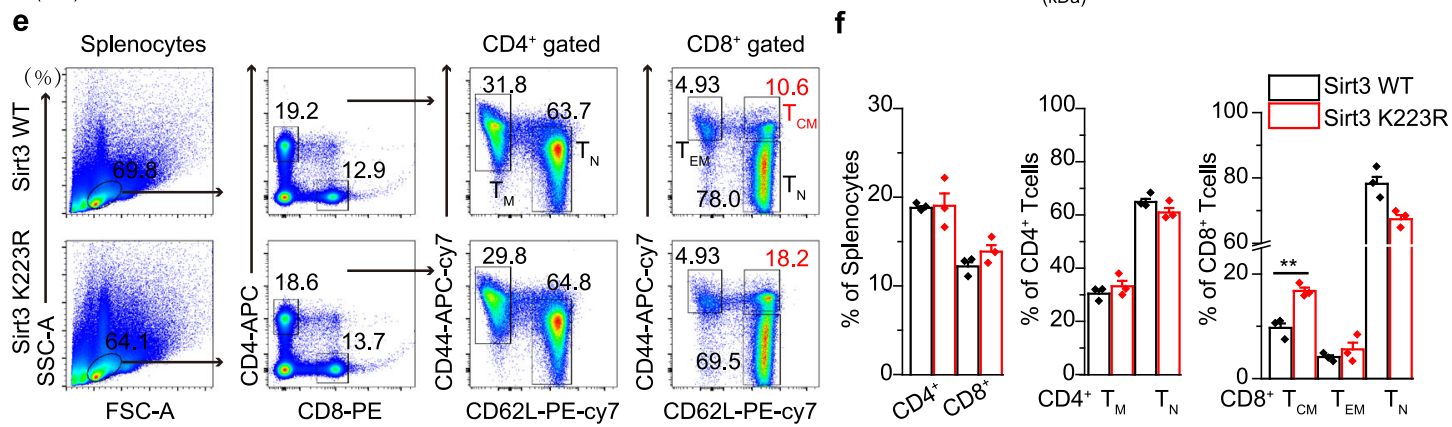

g
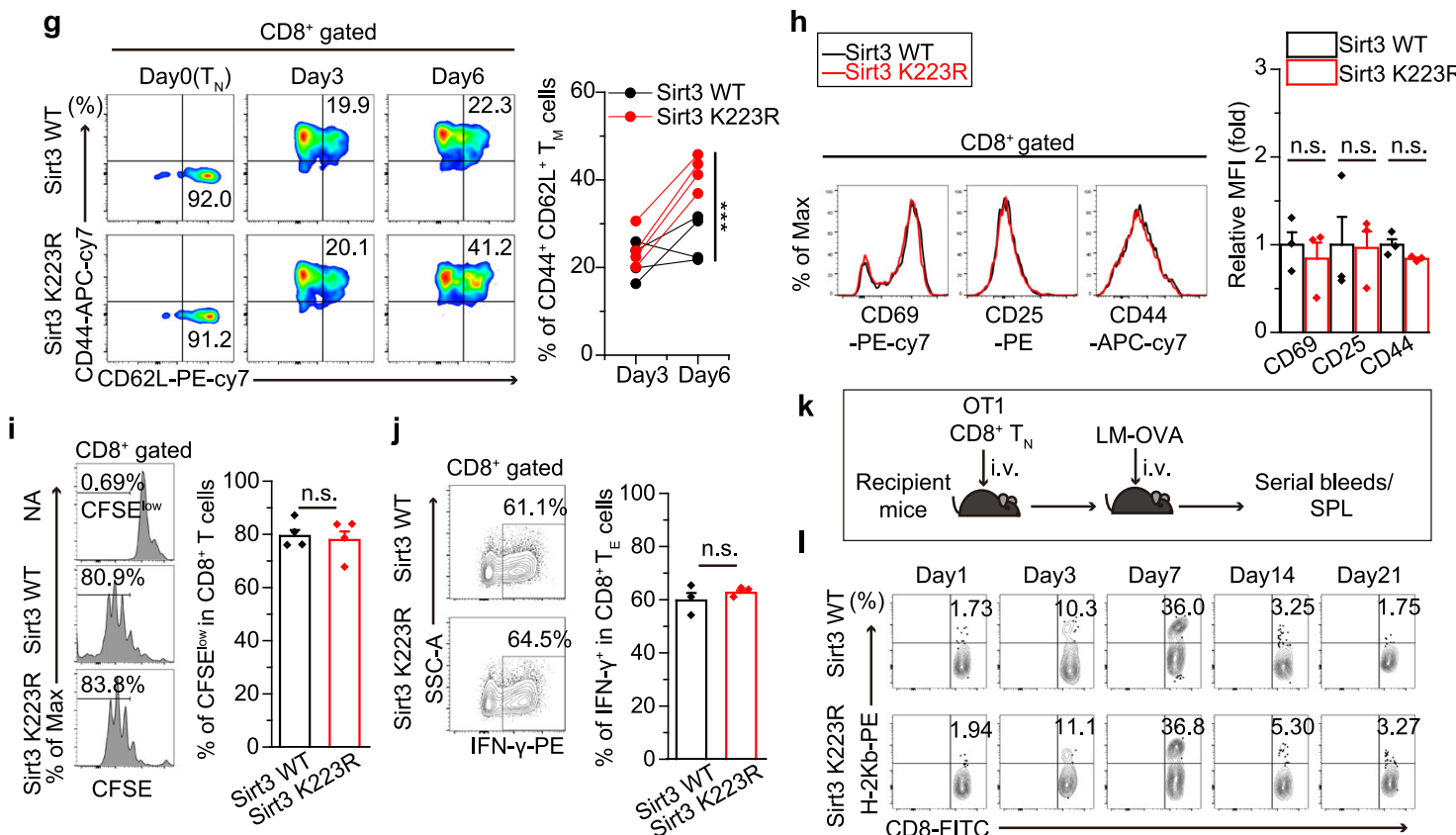

j
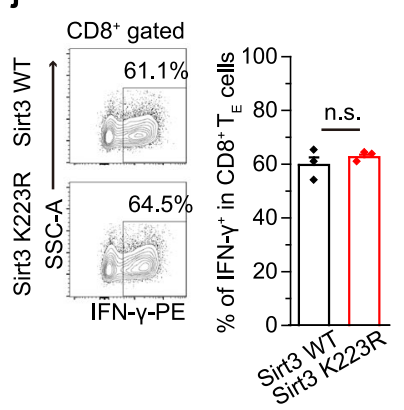

k

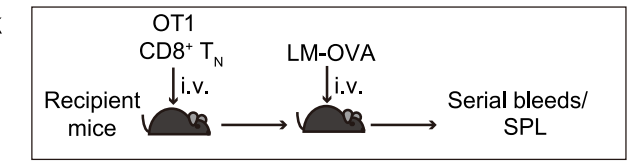

m

n
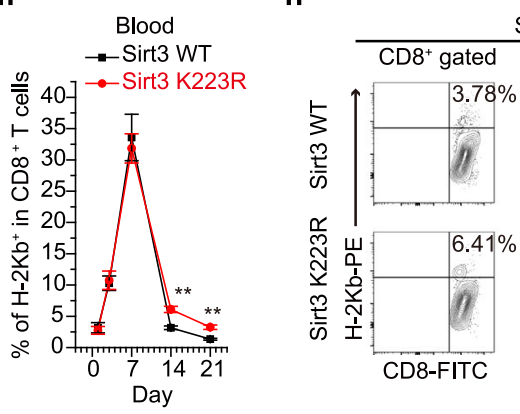

SPL

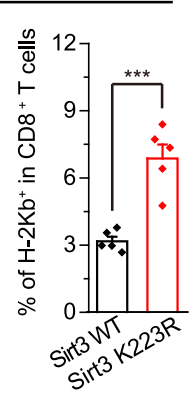

○

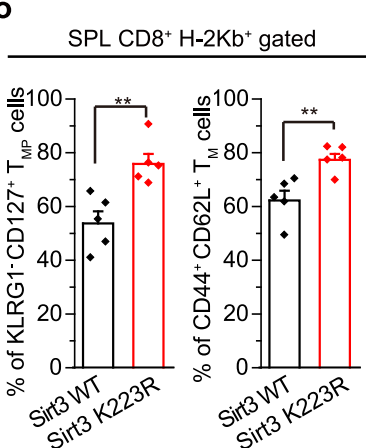

p

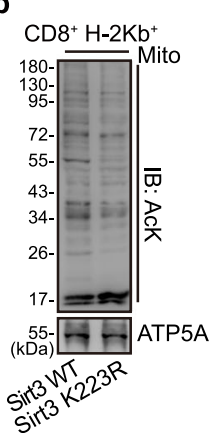

$\mathrm{T}$ cells were cultured in vitro and activated by $\mathrm{OVA}_{257-264}$ plus IL-2. We detected that the memory population $\left(\mathrm{CD}^{+} \mathrm{CD} 44^{+}\right.$ $\mathrm{CD} 2 \mathrm{~L}^{+}$) in Sirt3 K223R OT1 mice was significantly higher than that in Sirt3 WT mice in 6 days after $\mathrm{OVA}_{257-264}$ treatment (Fig. 1g). However, there were no significant changes between Sirt3 K223R and Sirt3 WT in the expression of T cell early activation markers (CD69, CD25 and CD44), T cell proliferation
(CFSE assay) and IFN- $\gamma$ production in OT1 $\mathrm{CD}^{+} \mathrm{T}$ cells treated by OVA $_{257-264}$ for 3 days (Fig. $1 \mathrm{~h}-\mathrm{j}$ ). These data suggest SENP1Sirt3 axis predominantly engaged in $\mathrm{T}$ cell memory development but not in effector $\mathrm{T}$ cells in response to antigen stimulation. Moreover, adoptive transferred mice were infected with Listeria monocytogenes to confirm $\mathrm{T}$ cell memory development in vivo. $\mathrm{CD}^{+}{ }^{+}$naive $\mathrm{T}$ cells from Sirt3 WT-OT1 and Sirt3 K223R-OT1 
Fig. 1 Activation of SENP1-Sirt3 axis promotes $\mathbf{T}$ cell memory development. a The global acetylation of mitochondrial proteins in $C D 8^{+}$effector $T\left(T_{E}\right)$ and memory $T\left(T_{M}\right)$ cells $\left(n=4\right.$ biologically independent samples). b Sirt3 SUMOylation of $T_{E}$ and $T_{M}$ cells. c SENP1 protein in total cell lysates (TCLs) and mitochondrial lysates (mito) of $T_{E}$ and $T_{M}$ cells. $\mathbf{d}$ The global acetylation of mitochondrial lysates from Senp1 WT and cKO CD8 ${ }^{+} T_{M}$ cells $(n=4$ biologically independent samples). e, f The $T_{M}$ and naïve $T\left(T_{N}\right)$ cells of $C D 4+T$ cell and the central memory $T\left(T_{C M}\right)$, effector-memory $T$ ( $\left.T_{E M}\right)$, and $T_{N}$ cells of $\mathrm{CD}^{+} \mathrm{T}$ cell in spleen were analysed $\left(n=3\right.$ mice, $\left.{ }^{\star \star} P=0.0059\right)$. g-h OT1 $\mathrm{T}_{\mathrm{N}}$ cells were activated with OVA $257-264$ peptide $+\mathrm{IL}-2$ for $3-6$ days. The population of $\mathrm{T}_{\mathrm{M}}$ cells were analysed $\left(n=4\right.$ mice, $\left.{ }^{\star \star \star} P=0.0004\right)(\mathbf{g})$. The indicated surface markers were analysed on day 3 ( $n=3$ mice) (h). $\mathbf{i}$ The percentages of CFSElow from activated OT1 $\mathrm{T}_{\mathrm{N}}$ cells $\left(n=4\right.$ mice), nonactivation (NA). $\mathbf{j}$ The ratios of IFN- $\gamma^{+}$cells in activated OT1 TN cells $(n=3$ mice). k-p OT1 $\mathrm{T}_{\mathrm{N}}$ cells were adoptive transferred into congenic recipient mice, and then the recipients were injected with LM-OVA ( $n=5$ mice) $(\mathbf{k})$. The percentage of $\mathrm{H}-2 \mathrm{~Kb}^{+} \mathrm{CD} 8+\mathrm{T}$ cells in the blood of recipients ( ${ }^{\star \star} P=0.0017$ on day 14 and ${ }^{\star \star} \mathrm{P}=0.0037$ on day 21$)(\mathbf{I}, \mathbf{m})$. The percentage of $\mathrm{H}-2 \mathrm{~Kb}+$ in the spleen $\mathrm{CD}^{+}{ }^{+} \mathrm{T}$ cells of recipients on day $\left.21{ }^{\star \star \star} \mathrm{P}=0.00048\right)(\mathbf{n})$. The population of memory precursor $\left(\mathrm{T}_{\mathrm{MP}}\right)$ and $\mathrm{T}_{\mathrm{M}}$ cells in the splenic $\mathrm{CD} 8^{+} \mathrm{H}_{-}$ $2 \mathrm{~Kb}^{+}$cells of recipients on day $21{ }^{\star \star} P=0.0055$ in $T_{M P}$, and ${ }^{\star \star} P=0.0076$ in $\left.T_{M}\right)(\mathbf{o})$. The global acetylation of mitochondrial proteins in the splenic $C D 8+$ $\mathrm{H}-2 \mathrm{~Kb}^{+}$cells from recipients (p). All immunoblots are representative of three independent experiments (b, $\left.\mathbf{c}, \mathbf{p}\right)$. Two-tailed unpaired $t$-test (f, $\left.\mathbf{n}, \mathbf{o}\right)$. Twoway ANOVA followed by Fisher's LSD test $(\mathbf{g}, \mathbf{m})$. All data are shown as the means \pm SEMs. n.s. not significant.

(donor cells) were adoptively transferred into congenic recipient mice, and then the recipients were injected (i.v.) with Listeria monocytogenes expressing-OVA (LM-OVA) ${ }^{14,41}$ (Fig. 1k). By serially bleeding and the detection of antigen-specific $\mathrm{CD}^{+}$ $\mathrm{T}$ cells $\left(\mathrm{OT} 1\right.$ tetramer ${ }^{+}, \mathrm{H}-2 \mathrm{~Kb}^{+}$) in the blood of recipients, we found that both Sirt3 WT-OT1 and Sirt3 K223R-OT1 $\mathrm{T}_{\mathrm{N}}$ cells mounted strong effector responses (day 3-7) (Fig. $1 \mathrm{k}-\mathrm{m}$ and Supplementary Fig. 1h). However, the percentage of $\mathrm{H}-2 \mathrm{~Kb}^{+}$cells from Sirt3 K223R-OT1 donors was higher than that from Sirt3 WT-OT1 donors at day 14 to 21 after infection (Fig. $1 \mathrm{k}-\mathrm{m}$ and Supplementary Fig. $1 \mathrm{~h}) . \mathrm{H}-2 \mathrm{~Kb}^{+}$cells in the spleen of Sirt3 K223R-OT1 recipients were also more than those in Sirt3 WTOT1 at day 21 post-infection (Fig. 1n and Supplementary Fig. 1i), indicating that Sirt $3 \mathrm{~K} 223 \mathrm{R}$ could promote T memory development. We further showed more $\mathrm{T}$ memory precursor cells $\left(\mathrm{T}_{\mathrm{MP}}\right.$, $\mathrm{KLRG}^{-} \mathrm{CD} 127^{+}$) and $\mathrm{T}$ memory cells $\left(\mathrm{T}_{\mathrm{M}}, \mathrm{CD} 44^{+} \mathrm{CD} 62 \mathrm{~L}^{+}\right)$in the $\mathrm{CD}^{+} \mathrm{H}-2 \mathrm{~Kb}^{+}$splenocytes of Sirt3 K223R-OT1 recipients as compared to that of Sirt3 WT-OT1 recipients at day 21 after LMOVA infection (Fig. 1o and Supplementary Fig. 1i). Moreover, we also detected lower mitochondrial acetylation in Sirt3 K223R $\mathrm{CD}^{+} \mathrm{H}-2 \mathrm{~Kb}^{+}$cells as compared to that in Sirt3 WT CD8 ${ }^{+}$ $\mathrm{H}-2 \mathrm{~Kb}^{+}$cells (Fig. 1p). These results reveal that Sirt3 K223R can promote $\mathrm{T}$ cell memory development in vivo.

Glucose limitation activates SENP1-Sirt3 axis via AMPK in T cell memory T cells. Next, we would seek the upstream regulator for SENP1-Sirt3 activation during $\mathrm{T}$ cell memory development. Since AMPK signalling promotes memory $\mathrm{T}$ cell generation ${ }^{14,16,23}$, we thus speculated that AMPK would trigger the activation of SENP1-Sirt3 axis during memory development. Indeed, we observed that an AMPK agonist metformin treatment increased the mitochondrial SENP1, decreased the SUMOylated Sirt3, and reduced mitochondrial acetylation in T cells (Fig. 2a-d, and Supplementary Fig. 2a), suggesting that AMPK is an upstream regulator to trigger SENP1-Sirt3 signalling in T cells.

We further explored the physiological relevance of AMPK activation of SENP1-Sirt3 axis in T cells. It is known that the clonal expansion of effector $\mathrm{T}$ cells accelerates glucose consumption in effector sites, which results in glucose limitation at the late phase of immune response ${ }^{16,22}$. Further investigation showed that glucose limitation activated SENP1-Sirt3 signalling in mitochondria ${ }^{31,32}$. Therefore, we reasoned that glucose limitation would be a physiological signal to trigger AMPK coupled SENP1Sirt3 signalling during effector-memory $\mathrm{T}$ cell differentiation. To testify this, culture medium containing $2 \mathrm{mM}$ glucose was applied to mimic low-glucose condition ${ }^{42}$, and low glucose markedly increased the memory cell population in effector $\mathrm{T}$ cell culture condition at day 6 upon antigen stimulation (Fig. 2e and Supplementary Fig. 2b, c). Moreover, low glucose activated AMPK in effector T cells shown as increased phosphorylation of the AMPK substrate acetyl-CoA carboxylase (ACC) (Fig. 2f). Furthermore, low glucose induced mitochondrial SENP1, reduced mitochondrial Sirt3 SUMOylation and decreased mitochondrial protein acetylation in $\mathrm{CD}^{+}$effector $\mathrm{T}$ cells (Fig. 2f, g). Importantly, Compound $\mathrm{C}$, an AMPK inhibitor, reversed these low-glucose-triggered activates in effector $\mathrm{T}$ cells (Fig. 2e-g), suggesting that the glucose limitation triggers SENP1-Sirt3 signalling via AMPK to promote $\mathrm{T}$ effector-memory differentiation.

FBP suppresses AMPK coupled SENP1-Sirt3 signaling in memory T cells. AMPK is capable of sensing glucose availability and regulates metabolic adaption and effector responses ${ }^{42}$. In the early stage of immune response, glucose is available at effector site, which maintains low AMPK activation in these earlyactivated effector cells. The effectors would suffer from limitation of glucose and the other nutrients at effector site in the late phase of immune response, which activates AMPK in these late effectors $^{16,22}$. Interestingly, it has been demonstrated that late effector $\mathrm{T}$ cells exhibit much lower glycolysis activity than the early effectors $\mathrm{do}^{22,43}$, suggesting that the production of glycolytic metabolites such as fructose-1,6-bisphosphate (FBP) might be decreased in the late phase of immune response. In fact, we showed that FBP production in T cells cultured in low glucose containing medium markedly reduced (Supplementary Fig. 2e). Since FBP has been shown to repress AMPK activation in glucose starvation $^{42}$, we proposed that glucose limitation may trigger AMPK coupled SENP1-Sirt3 signaling via reducing FBP production during effector-memory $\mathrm{T}$ cell differentiation. To prove this hypothesis, the effector $\mathrm{T}$ cells were cultured in regular or low glucose containing media supplemented with FBP $(5 \mathrm{mM})$ for 3 days (Supplementary Fig. 2d-f) ${ }^{44,45}$. FBP addition markedly reduced the low glucose effects on AMPK activation via an AMP/ ADP-independent mechanism (Supplementary Fig. 2f), and inhibited memory development from Sirt3 WT effector T cells (Fig. 2h). Notably, FBP had no effect on memory development from Sirt3 K223R $\mathrm{T}_{\mathrm{E}}$ cells even under low-glucose condition (Fig. 2h, Supplementary Fig. 2g). We further determined whether FBP affect the AMPK coupled SENP1-Sirt3 signalling in T cells. As shown in Fig. 2i, FBP blocked low-glucose-induced AMPK phosphorylation. FBP treatment also prevented low-glucoseinduced mitochondrial SENP1 level and reduction of Sirt3 SUMOylation in cultured CD8 ${ }^{+} \mathrm{T}_{\mathrm{E}}$ cells. Low-glucose-induced mitochondrial protein deacetylation was also reversed in FBP treated CD8 ${ }^{+} \mathrm{T}_{\mathrm{E}}$ cells (Fig. $2 \mathrm{i}, \mathrm{j}$ ).

To further determine the role of SENP1-Sirt3 signaling in AMPK-mediated $\mathrm{T}$ memory development, we analysed $\mathrm{T}$ memory cell development in Senp1 cKO T cells. Senp1 deficiency decreased $\mathrm{T}$ memory cell development under normal glucose condition. Low glucose or metformin treatment could not 
a

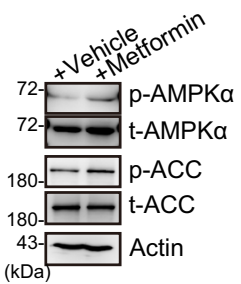

b

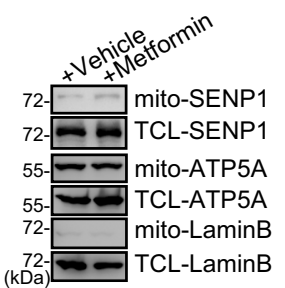

C

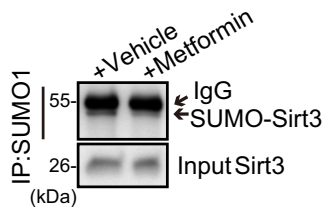

d

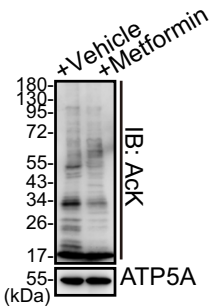

e

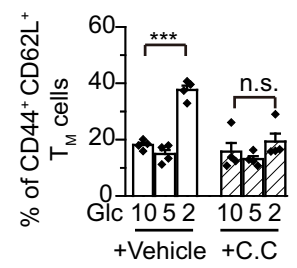

i

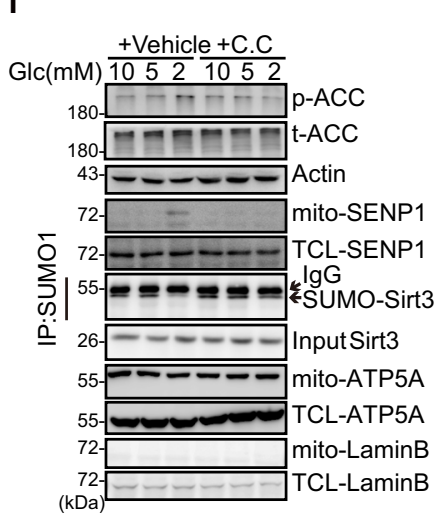

\section{g}

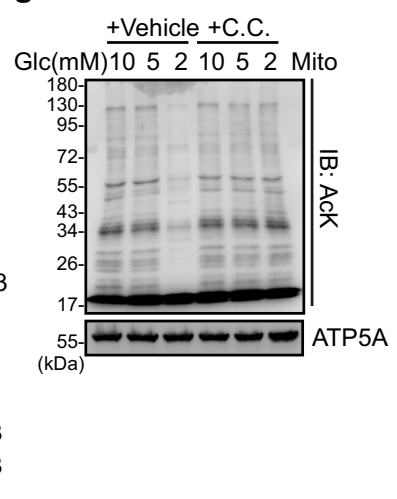

h

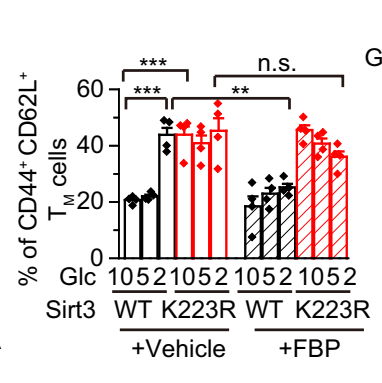

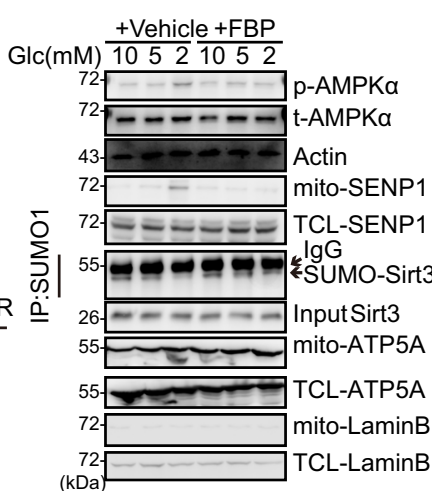

j

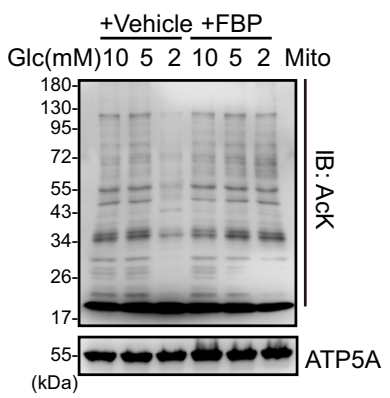

n

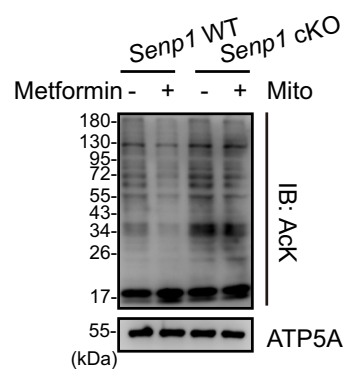

$\mathbf{k}$

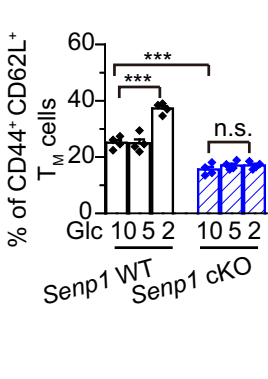

o
I

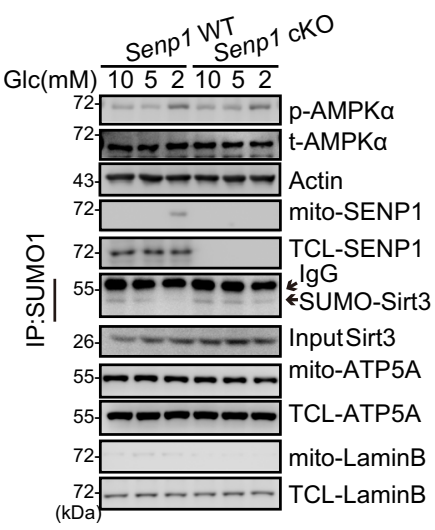

p m

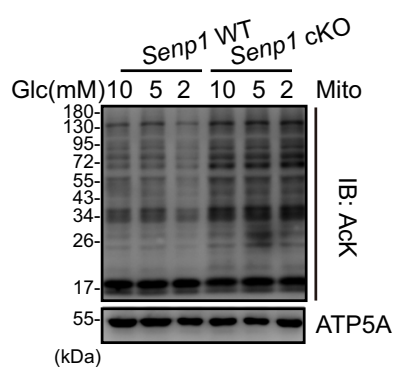

q increase the population of Senp1 cKO T memory cells as compared to Senp1 WT (Fig. 2k and Supplementary Fig. 2h-j). Moreover, we did not observe a change in SUMOylated Sirt3 as well as mitochondrial acetylation in the mitochondria of Senp1

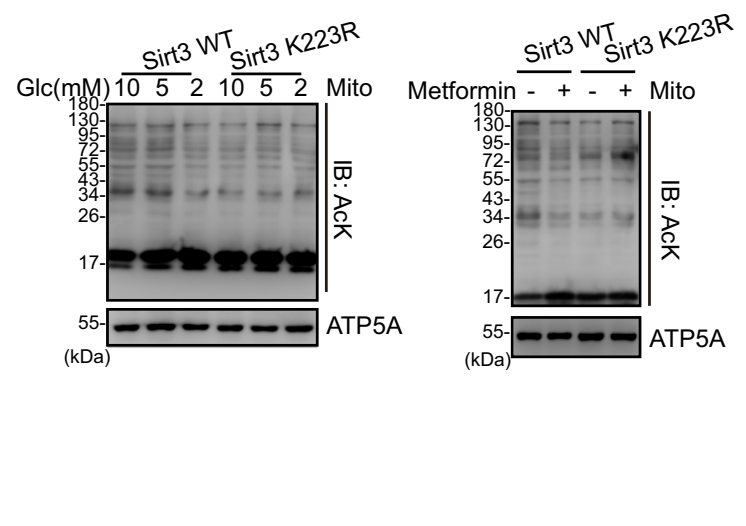

cKO T cells responding to low glucose or metformin treatment as compared to Senp1 WT control T cells (Fig. 2l-n). However, the mitochondrial acetylation in Sirt3 K223R CD8 ${ }^{+} \mathrm{T}$ cells was lower than that in Sirt3 WT control cells, and was maintained under 
Fig. 2 AMPK-SENP1-Sirt3 axis in T cell memory development. a-d The AMPK/ACC phosphorylation (a), mitochondrial SENP1 (b), Sirt3 SUMOylation (c), and the mitochondrial acetylation level (d) in activated $T_{N}$ cells treated with metformin ( $2 \mathrm{mM}, 3$ days). e-g Activated $T_{N}$ cells cultured with glucose (Glc, 10, 5 and $2 \mathrm{mM}$ ) and compound C (C.C, $10 \mu \mathrm{M}, 3$ days). The populations of $\mathrm{T}_{\mathrm{M}}$ cells ( $n=4$ biologically independent samples, $\left.{ }^{\star \star \star} P<0.0001\right)$ (e), the AMPK phosphorylation, mitochondrial SENP1, Sirt3 SUMOylation and the mitochondrial acetylation level were detected (f, $\mathbf{g})$. h-j Activated $T_{N}$ cells cultured with Glc (10, 5 and $2 \mathrm{mM}$ ) and fructose-1,6-bisphosphate (FBP, $5 \mathrm{mM}, 3$ days). The populations of $\mathrm{T}_{\mathrm{M}}$ cells ( $n=4$ biologically independent samples, ${ }^{\star \star \star} P<0.000110$ vs $2 \mathrm{mM} \mathrm{Glc}$ culture in vehicle treated WT cells, ${ }^{\star \star \star} P<0.0001$ Sirt3 WT vs K223R cells in $10 \mathrm{mM}$ Glc and vehicle, ${ }^{\star \star} P=0.003$ vehicle vs FBP treatment in $2 \mathrm{mM} \mathrm{Glc}$ cultured WT cells) (h), the AMPK phosphorylation, mitochondrial SENP1, Sirt3 SUMOylation and the mitochondrial acetylation level were detected $(\mathbf{i}, \mathbf{j})$. $\mathbf{k}-\mathbf{m}$ Activated $T_{N}$ cells cultured with Glc $\left(10,5\right.$ and $2 \mathrm{mM}$ ). The populations of $T_{M}$ cells ( $n=4$ biologically independent samples, ${ }^{* \star \star} P<0.000110$ vs $2 \mathrm{mM}$ Glc culture in WT cells, ${ }^{\star \star \star} P=0.0003$ Senp1 WT vs cKO cells in $10 \mathrm{mM}$ Glc culture) (k), the AMPK phosphorylation, mitochondrial SENP1, Sirt3 SUMOylation and the mitochondrial acetylation level were detected (I, m). n The mitochondrial acetylation level of activated Senp1 WT and cKO T $T_{N}$ cells with vehicle or metformin ( 2 mM, 3 days). o, p Activated Sirt3 WT and K223R OT1 TN cells cultured with Glc (10, 5 and $2 \mathrm{mM}$ ). The AMPK phosphorylation, mitochondrial SENP1, Sirt3 SUMOylation and the mitochondrial acetylation level were detected. $\mathbf{q}$ Activated Sirt3 WT and K223R OT1 $T_{N}$ cells cultured with vehicle or metformin ( $2 \mathrm{mM}, 3$ days). The mitochondrial acetylation level was detected. All immunoblots are representative of three independent experiments $(\mathbf{a}-\mathbf{d}, \mathbf{f}, \mathbf{g}, \mathbf{i}, \mathbf{j}, \mathbf{I}-\mathbf{q})$. Two-way ANOVA followed by Fisher's LSD test (e, h, $\mathbf{k})$. All data are shown as the means \pm SEMs. n.s. not significant.

low glucose or metformin treatment (Fig. 2o-q). These data suggest that glucose limitation may trigger AMPK coupled SENP1-Sirt3 signalling in T cell memory development.

SENP1-Sirt3 axis enhances the survival of memory T cells. We next explored the mechanism underlying SENP1-Sirt3 axis promoting $\mathrm{T}$ cell memory development. It is well-known that cell survival is a critical feature gained for $\mathrm{T}$ cell memory development ${ }^{46,47}$. There were much less apoptotic central memory $\mathrm{T}$ cells $\left(\mathrm{T}_{\mathrm{CM}}, \mathrm{CD}{ }^{+} \mathrm{CD} 2 \mathrm{~L}^{+} \mathrm{CD} 44^{+}\right)$in the spleens of Sirt3 K223R mutant mice than that of Sirt3 WT mice (Fig. 3a, b), suggesting that SENP1-Sirt3 axis may regulate $T_{M}$ cell survival. In vivo survival assay using $\mathrm{T}$ cell transplantation model also showed that the Sirt3 $\mathrm{K} 223 \mathrm{R}$ donor $\mathrm{T}_{\mathrm{M}}$ cells in the spleens (SPL) or lymph nodes (LNs) of the recipient mice were markedly more abundant than Sirt3 WT donor $\mathrm{T}_{\mathrm{M}}$ cells in the recipient mice (Fig. 3c). Additionally, Sirt3 K223R $\mathrm{T}_{M}$ cells exhibited a higher mitochondrial membrane potential (MMP), an indicator of mitochondrion-dependent apoptosis, than Sirt3 WT $\mathrm{T}_{\mathrm{M}}$ cells did (Fig. 3d), suggesting that SENP1-Sirt3 axis may protect mitochondria against apoptotic challenges. Moreover, treating $\mathrm{T}_{M}$ cells with the sirtuin inhibitor nicotinamide (NAM) (Fig. 3e) or the knockout of Sirt3 in $\mathrm{T}_{M}$ cells markedly decreased the in vitro survival of $\mathrm{T}_{M}$ cells (Fig. $3 \mathrm{f}$ and Supplementary Fig. 3a). Consistently, Senp1 cKO $\mathrm{T}_{M}$ cells also decreased survival compared with Senp1 WT cells (Fig. 3g).

We generated an OT1 $\mathrm{T}$ cell transplantation model to investigate the role of SENP1-Sirt3 axis in $\mathrm{T}$ cell survival in vivo. Sirt3 WT or Sirt3 K223R OT1 tetramer ${ }^{+}\left(\mathrm{H}-2 \mathrm{~Kb}^{+}\right)$ $\mathrm{T}$ cells were transplanted into congenic recipient mice (Supplementary Fig. 3b, c). The population of Sirt3 K223R CD8 ${ }^{+}$ $\mathrm{H}-2 \mathrm{~Kb}^{+} \mathrm{T}$ cells was slightly larger than that of Sirt3 WT CD8 ${ }^{+}$ $\mathrm{H}-2 \mathrm{~Kb}^{+} \mathrm{T}$ cells at day 7 post-transplantation. However, much more abundance of Sirt3 K223R CD8 ${ }^{+} \mathrm{H}-2 \mathrm{~Kb}^{+} \mathrm{T}$ cells (assumed memory $\mathrm{T}$ cells) than Sirt $3 \mathrm{WT} \mathrm{CD}^{+} \mathrm{H}-2 \mathrm{~Kb}^{+} \mathrm{T}$ cells was observed in the recipient mice at day 28 post-transplantation (Fig. 3h, i). Furthermore, Sirt3 K223R CD8 ${ }^{+} \mathrm{H}-2 \mathrm{~Kb}^{+}$splenocytes showed much less apoptotic ratio $\left(\right.$ Annexin $\mathrm{V}^{+}$) than Sirt3 WT $\mathrm{CD}^{+} \mathrm{H}-2 \mathrm{~Kb}^{+}$splenocytes did at day 21 after LM-OVA infection (Fig. 3j, k). Additionally, Sirt3 K223R OT1 $\mathrm{T}_{\mathrm{M}}$ cells displayed more IFN- $\gamma$ - and Granzyme B-positive T cells than Sirt3 WTOT1 $\mathrm{T}_{\mathrm{M}}$ cells upon $\mathrm{OVA}_{257-264}$ peptide re-challenge (Fig. $31, \mathrm{~m}$, and Supplementary Fig. $3 \mathrm{~d}-\mathrm{f}$ ). Thus, we propose that SENP1Sirt3 axis may be a positive signalling pathway that promotes $\mathrm{T}$ cell survival leading memory development.

SENP1-Sirt3 axis promotes OXPHOS and mitochondrial fusion in $\mathbf{T}_{\mathbf{M}}$ cells. To understand the metabolic basis of SENP1-
Sirt3 axis in promoting the survival of memory $\mathrm{T}$ cells, we measured the oxygen consumption rate (OCR) and the extracellular acidification rate (ECAR) in $T_{M}$ cells. Sirt3 K223R $T_{M}$ cells displayed a higher OCR but a lower ECAR than Sirt3 WT $\mathrm{T}_{\mathrm{M}}$ cells did (Fig. 4a, b). Consistently, OCR in Senp1-deficient $\mathrm{T}_{\mathrm{M}}$ cells was lower than that in Senp1 WT $\mathrm{T}_{\mathrm{M}}$ cells (Fig. 4c). In addition, Sirt3 K223R $\mathrm{T}_{M}$ cells had fewer long-chain acylcarnitine species and higher levels of ATP and Acetyl-CoA in mitochondria than Sirt3 WT $\mathrm{T}_{M}$ cell mitochondria (Supplementary Fig. 4a-c). These data suggest that the SENP1-Sirt3 signalling may promote FAO-fuelled OXPHOS in $\mathrm{T}_{M}$ cells.

Mitochondrial fusion is required for the increase in OXPHOS activity linked to $T_{M}$ cell development ${ }^{7}$. Thus, we tested whether the SENP1-Sirt3 axis promotes mitochondrial fusion in $T_{M}$ cells. As shown in Fig. 4d, f, Sirt3 K223R $\mathrm{T}_{M}$ cells presented increased mitochondrial mass and larger mitochondrial area as compared to Sirt3 WT $\mathrm{T}_{\mathrm{M}}$ cells. Senp1 deficiency reduced the mitochondrial mass in $\mathrm{T}_{\mathrm{M}}$ cells compared with Senp1 WT accordingly (Fig. 4e). Furthermore, the mitochondrial size and surface area in Sirt3 K223R $\mathrm{T}_{\mathrm{M}}$ cells were higher than those in Sirt3 WT cells (Fig. 4gi). Sirt3 K223R $T_{M}$ cells showed more cristae per mitochondria and a decreased maximal cristae width than Sirt3 WT $\mathrm{T}_{M}$ cells (Fig. 4j, k). The mitochondrial mass and OCR were more increase in Sirt3 K223R CD8 ${ }^{+} \mathrm{H}-2 \mathrm{~Kb}^{+}$cells from the spleen of recipients than that in Sirt3 WT control at day 21 after LM-OVA infection (Fig. 4l-n). These data indicate that the SENP1-Sirt3 axis may enhance mitochondrial fusion and mitochondrial inner membrane remodelling in $\mathrm{T}_{M}$ cells.

YME1L1 is a substrate of Sirt3 in OPA1 processing and mitochondrial fusion. The mitochondrial inner membrane protein OPA1 is a master regulator of mitochondrial inner membrane fusion and cristae remodelling ${ }^{48-52}$. There are several OPA1 isoforms in mammalian cells. Long-form OPA1 (L-OPA1) is located in the inner membrane and promotes mitochondrial inner membrane fusion ${ }^{21,49}$. L-OPA1 is cleaved at two distinct sites to short-form OPA1 (S-OPA1), which is released into the intermembrane space, by two proteins called yeast mitochondrial AAA metalloprotease YME1L1 and metalloendopeptidase OMA1 ${ }^{19,48,49}$. Although S-OPA1 is dispensable for fusion, its accumulation promotes mitochondrial fission ${ }^{48}$. To determine whether the SENP1-Sirt3 axis modulates OPA1-mediated mitochondrial fusion in $\mathrm{T}_{M}$ cells, levels of OPA1 isoforms in Sirt3 K223R and WT cells were measured. We found that Sirt3 K223R $\mathrm{T}_{\mathrm{M}}$ cells had higher levels of mitochondrial L-OPA1 than Sirt3 WT cells (Fig. 5a), which indicated that the SENP1-Sirt3 axis suppresses OPA1 cleavage. 
a

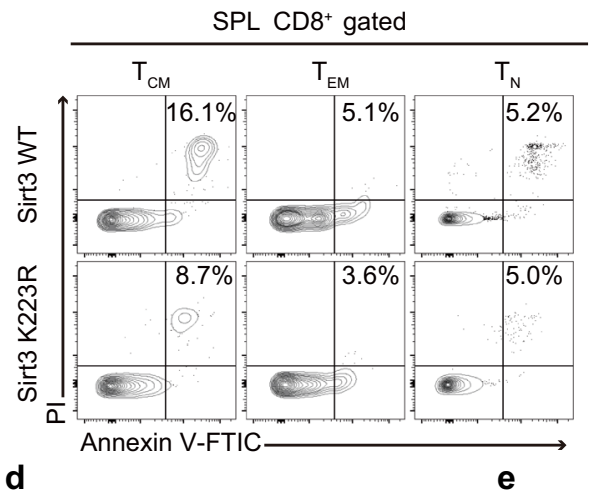

b

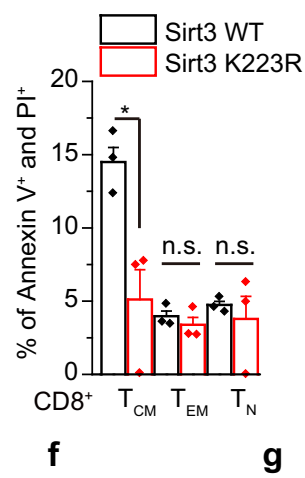

C

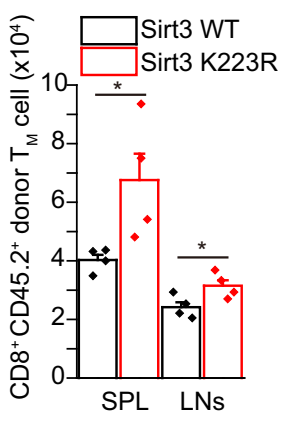

h i

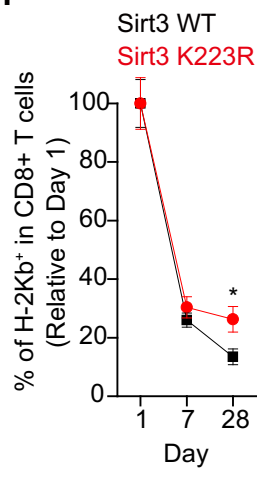

j

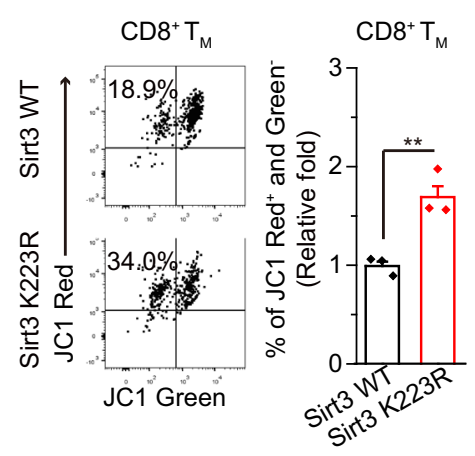

LM-OVA infected

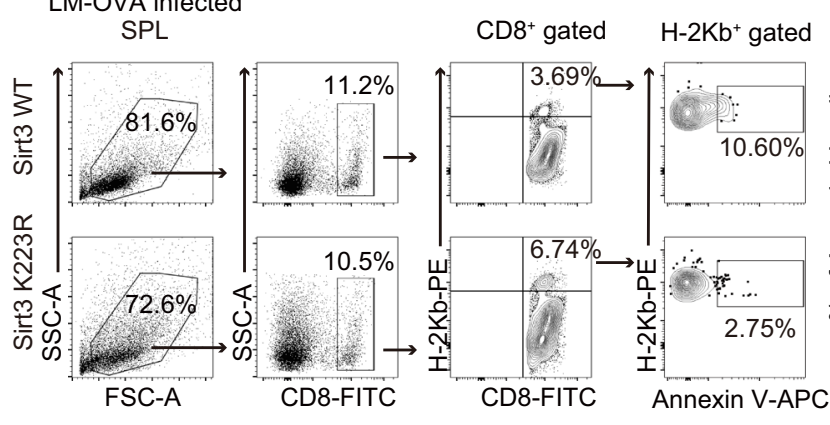

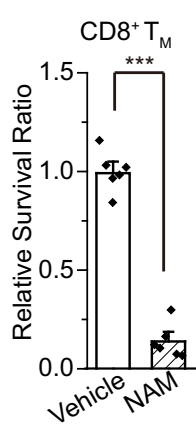

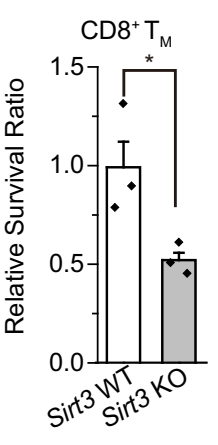

k

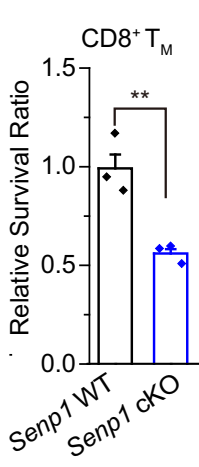

I

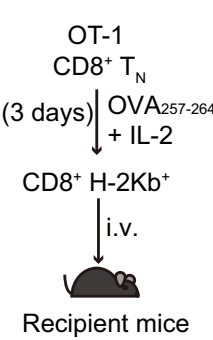

m
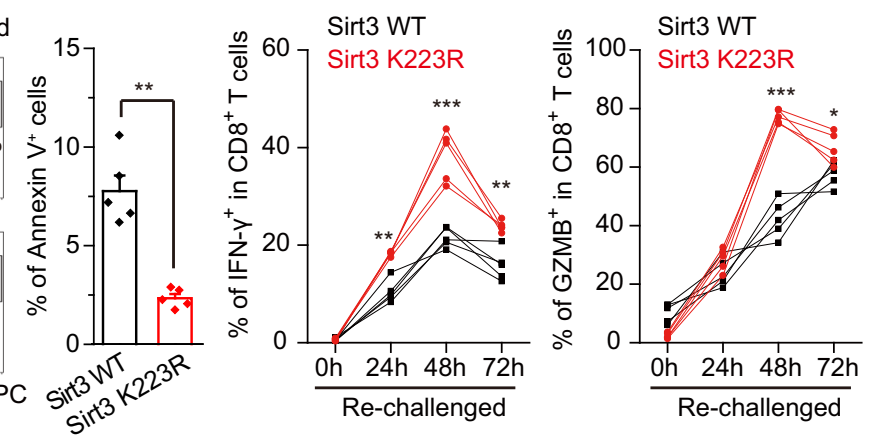

Fig. 3 SENP1-Sirt3 axis enhances the survival of $\mathbf{T}_{\mathbf{M}}$ cells. $\mathbf{a}$, $\mathbf{b}$ The apoptotic ratios of CD8 ${ }^{+} T_{C M}, T_{E M}$ and $T_{N}$ cells from Sirt3 WT or K223R mouse splenocytes were measured $\left(n=3\right.$ mice, $\left.{ }^{\star} P=0.028\right)$. c $T_{M}$ cells $\left(1.5 \times 10^{6}, C D 45.2\right)$ generated from Sirt3 WT or K223R mice were injected into congenic recipient mice (CD45.1). The donor $T_{M}$ cell numbers in the recipient spleen (SPL) or lymph nodes (LNs) were analysed on day $2\left(n=4\right.$ mice, ${ }^{\star} P=0.042$ in SPL and ${ }^{\star} P=0.046$ in LN). d T M cells generated from Sirt3 WT or K223R mice were analysed by JC- 1 staining. ( $n=3$ biologically independent samples, $\left.{ }^{\star \star} P=0.0083\right)$. e The survival ratio of $T_{M}$ cells treated with NAM $(5 \mathrm{mM})$ on day 3 was analysed $\left(n=6\right.$ biologically independent samples, $\left.{ }^{\star \star \star} P<0.0001\right)$. $\mathbf{f}$ The survival ratio of $\mathrm{T}_{M}$ cells from Sirt3 WT or Sirt3-KO mice was detected ( $n=3$ biologically independent samples, $\left.{ }^{\star} P=0.047\right)$. $\mathbf{g}$ The survival ratio of $\mathrm{T}_{\mathrm{M}}$ cells from Senp1 WT or cKO mice was analysed ( $n=3$ biologically independent samples, $\left.{ }^{\star \star} P=0.0089\right)$. $\mathbf{h}, \mathbf{i}$ The activated OT1 T cells $\left(\mathrm{H}-2 \mathrm{~Kb}{ }^{+}\right)$were transplanted into congenic recipient mice $\left(1.0 \times 10^{7} /\right.$ mouse $)(\mathbf{h})$. The $\mathrm{H}-2 \mathrm{~Kb}^{+}$populations in the recipient blood were analyzed $\left(n=7\right.$ mice, $\left.{ }^{\star} P=0.024\right)$ (i). j, $\mathbf{k}$ The apoptotic ratio of spleen $\mathrm{CD}^{+} \mathrm{H}-2 \mathrm{~Kb}^{+}$cells from recipients was quantified after 21 days LM-OVA infection in vivo $\left(n=5\right.$ mice, ${ }^{\star \star} P=$ 0.0016).). I, $\mathbf{m}$ The populations of IFN- $\gamma(\mathbf{I})$ - or Granzyme B (GZMB) ( $\mathbf{m})$-positive T cells in $\mathrm{T}_{\mathrm{M}}$ cells were analysed after re-challenge $\left(n=5\right.$ mice, ${ }^{\star \star} P=$ $0.0013,{ }^{* \star \star} P \leq 0.001,{ }^{\star \star} P=0.0033$ at 24,48 and $72 \mathrm{~h}$, respectively, for IFN- $\gamma ;{ }^{\star \star \star} P \leq 0.0001$ and ${ }^{\star} P=0.029$ at $48 \mathrm{~h}$ and $72 \mathrm{~h}$, respectively, for GZMB). Two-tailed unpaired $t$-test $(\mathbf{b}-\mathbf{g}, \mathbf{k})$. Two-way ANOVA followed by Fisher's LSD test $(\mathbf{i}, \mathbf{I}, \mathbf{m})$. All data are shown as the means \pm SEMs. n.s. not significant.

Sirt3 can interact with YME1L1 but not OMA1 ${ }^{53}$ (Fig. 5b). We further identified YME1L1 as an acetylated protein (Supplementary Fig. 5a) and thus hypothesized that Sirt3 might deacetylate YME1L1 to modulate OPA1 cleavage. NAMtreated Jurkat $\mathrm{T}$ cells or Sirt3-knockout $\mathrm{T}_{\mathrm{M}}$ cells increased YME1L1 acetylation (Fig. 5c, d). Sirt3 K223R $\mathrm{T}_{\mathrm{M}}$ cells reduced YME1L1 acetylation as compared to Sirt3 WT cells (Fig. 5e). Furthermore, we mapped the K237 site as a unique acetylation residue on YME1L1 (Fig. 5f and Supplementary Fig. 5a). The
K237R mutant impaired the ability of YME1L1 to process OPA1 cleavage (Fig. $5 \mathrm{~g}$ ) and subsequently promoted mitochondrial fusion (Fig. 5h-k). In contrast, YME1L1 K237Q mutant mimicking its acetylated form promoted OPA1 processing, resulted in a decrease in mitochondrial fusion compared with YME1L1 WT and K237R (Fig. 5h-k, and Supplementary Fig. 5b). These data reveal that Sirt3 deacetylates YME1L1 leading to suppression of OPA1 processing, which promotes mitochondrial fusion. 
a

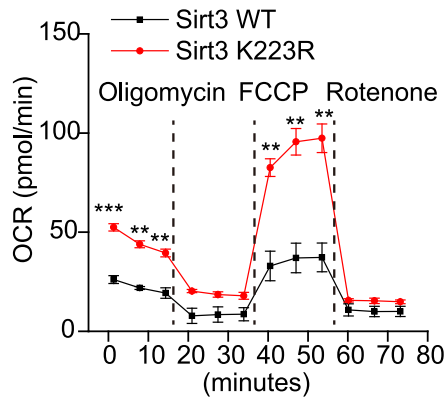

b

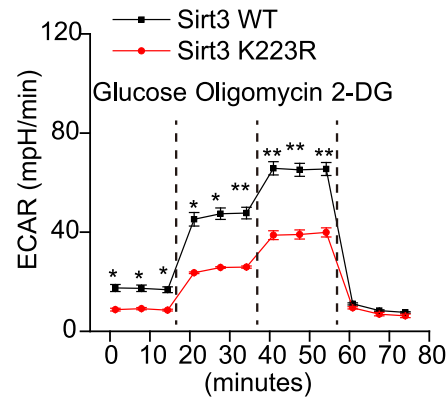

C

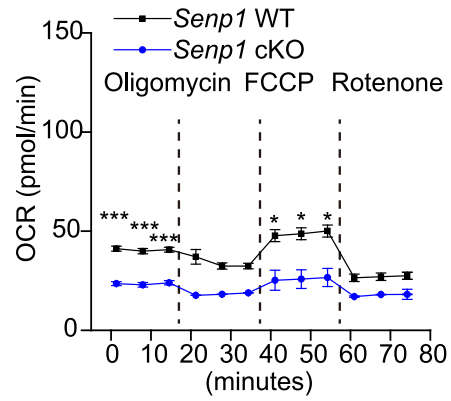

d

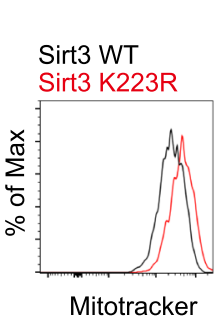

g

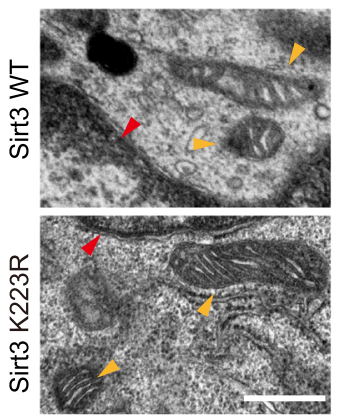

e

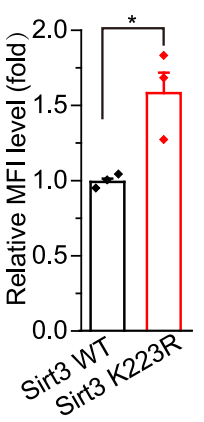

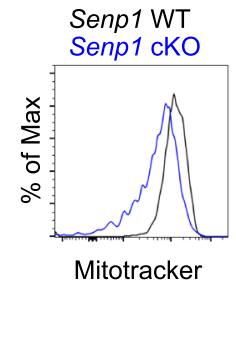

f

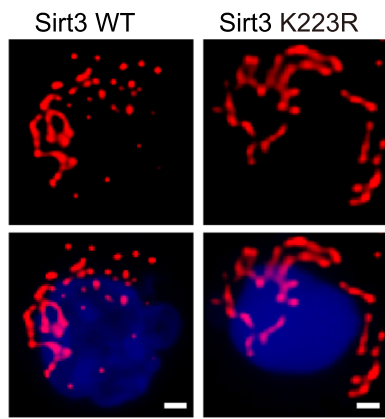

i

j

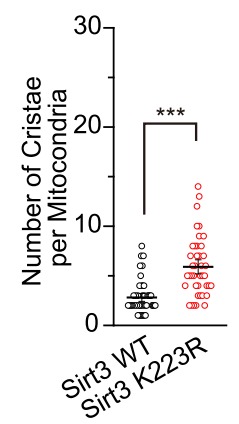

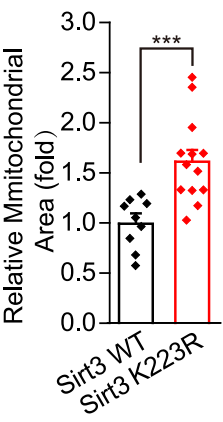

k

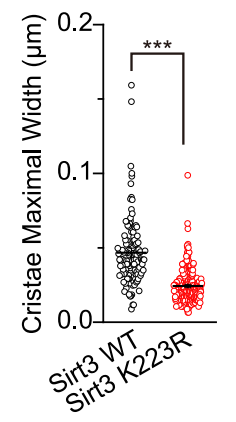

I LM-OVA infected

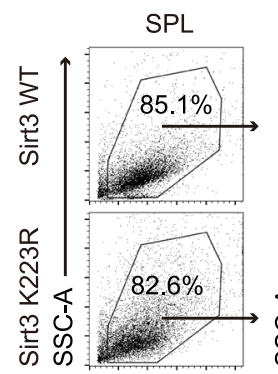

FSC-A

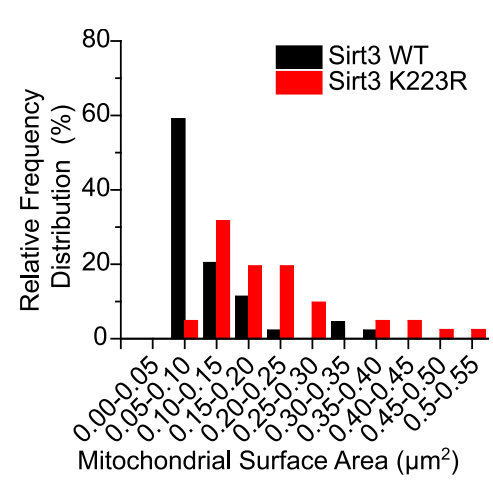

Mitochondrial Surface Area $\left(\mu \mathrm{m}^{2}\right)$

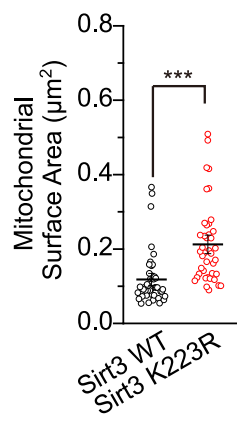

m

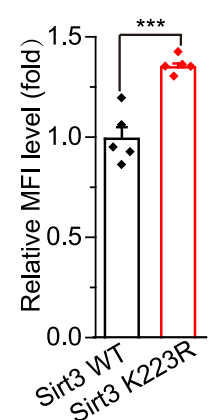

n

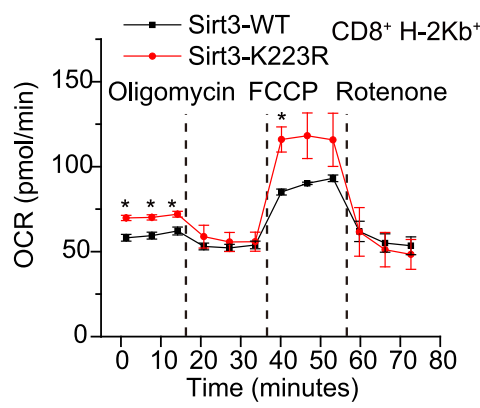

To determine whether YME1L1 acetylation affects $T_{M}$ cell survival, we overexpressed YME1L1 mutants in either Sirt3 WT or K223R $\mathrm{T}_{\mathrm{M}}$ cells by lentiviral transduction. As shown in Fig. 5l, $\mathrm{m}$, YME1L1 K237R expression significantly reduced the apoptotic cell numbers in Sirt3 WT $\mathrm{T}_{M}$ cells but not in Sirt3 K223R $\mathrm{T}_{M}$ cells. In contrast, YME1L1 K237Q induced the apoptosis of both Sirt3 WT and K223R $\mathrm{T}_{\mathrm{M}}$ cells (Fig. 5l, m, and Supplementary Fig. 5c). These data indicate that the Sirt3-mediated deacetylation of YME1L1 promotes $\mathrm{T}_{M}$ cell survival and $\mathrm{T}$ cell memory development.
Sirt3 K223R enhances the anti-tumour immunity of $\mathrm{CD8}^{+}$ T cells. To explore the biological significance of SENP1-Sirt3 signalling in $\mathrm{T}_{\mathrm{M}}$ cells, we transplanted Sirt3 WT or K223R $\mathrm{T}_{M}$ $\left(\mathrm{OT} 1 / \mathrm{H}-2 \mathrm{~Kb}^{+}\right)$cells into congenic recipient mice. At day 7 posttransplantation, we inoculated MC38-OVA tumour cells into the recipient mice subcutaneously (Fig. 6a) and observed drastically delayed tumour growth in the Sirt3 K223R $\mathrm{T}_{\mathrm{M}}$ cell-transplanted mice compared with that in the Sirt $3 \mathrm{WT} \mathrm{T}_{\mathrm{M}}$ cell-transplanted mice (Fig. 6b, c). The analysis of tumour-infiltrating CD8 ${ }^{+} \mathrm{T}$ cells showed that the frequency of Sirt3 K223R donor CD8 ${ }^{+} \mathrm{T}$ cells 
Fig. 4 The SENP1-Sirt3 axis promotes OXPHOS and mitochondrial fusion in $\mathbf{T}_{\mathbf{M}}$ cells. $\mathbf{a}, \mathbf{b}$ The OCR (a) and ECAR (b) of Sirt3 WT or K223R $\mathrm{T}_{M}$ cells $\left(n=3\right.$ mice, ${ }^{\star \star \star} P=0.0006,{ }^{\star \star} P=0.0022,{ }^{\star \star} P=0.0046,{ }^{\star \star} P=0.0085,{ }^{\star \star} P=0.0044{ }^{\star \star} P=0.0042$ at $1.3,7.8,14.3,40.5,47.0$ and 53.5 min, respectively, (a); ${ }^{\star} P=0.015,{ }^{\star} P=0.016,{ }^{\star} P=0.013,{ }^{\star} P=0.015,{ }^{\star} P=0.01,{ }^{\star} P P=0.0093,{ }^{\star}{ }^{\star} P=0.002,{ }^{\star} P P=0.002{ }^{\star \star} P=0.0021$ at $1.3,7.9,14.4,21.1,27.7,34.2,40.9$, 47.5 and $54.2 \mathrm{~min}$, respectively (b). c The OCR of Senp1 WT or CKO TM cells $\left(n=3\right.$ mice, ${ }^{\star \star \star} P=0.0005,{ }^{\star \star \star} P=0.0007,{ }^{\star \star \star} P=0.0006,{ }^{\star} P=0.027,{ }^{\star} P=$ $0.02{ }^{\star} P=0.018$ at $1.3,7.9,14.6,41.1,47.7$ and 54.2 min, respectively). d, e The MitoTracker staining of TM cells from Sirt3 WT or K223R $\left(n=3\right.$ mice, ${ }^{\star} P=$ $0.025)(\mathbf{d})$, and Senp1 WT or cKO $\left(n=3\right.$ mice, $\left.{ }^{\star \star} P=0.0026\right)(\mathbf{e}) . \mathbf{f}$ The mitochondrial area of Sirt3 WT or K223R TM cells $(n=9$ cells for WT, $n=13$ cells for $\left.K 223 R,{ }^{\star \star \star} P=0.0008\right)$. The scale bars, $1 \mu \mathrm{m}$. $\mathbf{g}-\mathbf{k}$ The TEM of mitochondria in Sirt3 WT or K223R $\mathrm{T}_{\mathrm{M}}$ cells. The red arrows, nucleus. The orange arrows, mitochondria. The scale bars, $0.5 \mu \mathrm{m}(\mathbf{g})$. The mitochondrial surface area $\left(n=44\right.$ mitochondria from WT, $n=41$ mitochondria from K223R, $\left.{ }^{\star \star \star} P<0.0001\right)$ $(\mathbf{h}-\mathbf{i})$, the cristae number per mitochondria $\left({ }^{\star \star \star} P<0.0001\right)(\mathbf{j})$, and the maximal cristae width $\left(n=124\right.$ cristae from WT, $n=242$ cristae from K223R, ${ }^{\star \star \star} P$ $<0.0001)(\mathbf{k})$ were analyzed. I-n The MitoTracker staining $\left(n=5\right.$ mice, $\left.{ }^{\star \star \star} P=0.0004\right)(\mathbf{I}, \mathbf{m})$, the OCR $\left(n=3\right.$ biologically independent samples, ${ }^{\star} P=$ $0.011,{ }^{\star} P=0.016,{ }^{\star} P=0.031,{ }^{\star} P=0.047$ at $1.3,7.7,14.2$ and $40.2 \mathrm{~min}$, respectively) (n) of splenic CD8 ${ }^{+} \mathrm{H}-2 \mathrm{~Kb}{ }^{+}$cells after LM-OVA infection. Two-tailed unpaired $t$-test $(\mathbf{d}-\mathbf{f}, \mathbf{i}-\mathbf{k}, \mathbf{m})$. Two-way ANOVA followed by Fisher's LSD test $(\mathbf{a}-\mathbf{c}, \mathbf{n})$. All data are shown as the means \pm SEMs. n.s. not significant.

$\left(\mathrm{H}-2 \mathrm{~Kb}^{+}\right)$was higher than that of Sirt3 WT donor $\mathrm{CD}^{+} \mathrm{T}$ cells (Fig. 6d). Furthermore, we used OVA-activated OT1 T cells for immune therapy in the established MC38-OVA tumour model (Fig. 6e). As shown in Fig. 6f, g, Sirt3 K223R OT1 T cells were more potent in inhibiting tumour growth than Sirt3 WT-OT1 $\mathrm{T}$ cells (Fig. 6f, g). Consistently, Sirt3 K223R T cells $\left(\mathrm{H}-2 \mathrm{~Kb}^{+}\right)$ were markedly more abundant in MC38-OVA tumour tissue than Sirt3 WT T cells (Fig. 6h). These data suggest that activation of the SENP1-Sirt3 axis may potentially promote the anti-tumour immunity of T cells.

\section{Discussion}

In this study, we found that the AMPK coupling SENP1-Sirt3 signalling promotes $\mathrm{T}$ cell survival and memory development. Glucose limitation is a physiological signal to stimulate AMPK signalling and further activate SENP1-Sirt3 axis in T cell memory development. A mechanistic analysis revealed that SENP1-Sirt3 signalling promoted both OXPHOS activity and mitochondrial fusion through reducing YME1L1-mediated OPA1 cleavage in $\mathrm{T}_{\mathrm{M}}$ cells. Furthermore, we showed that glycolytic metabolite FBP suppressed AMPK coupled SENP1-Sirt3 signalling in $\mathrm{T}$ cells. Therefore, these data reveal that glucose limitation promotes AMPK-SENP1-Sirt3 signalling and T cell memory development via reducing FBP production (Fig. 6i).

Limitation of oxygen and nutrients can weaken or even damage the effector function in $\mathrm{T}$ cells. This study showed that glucose limitation would be beneficial for $\mathrm{T}$ cell memory development. Ma et al. analysed $\mathrm{T}$ cell glucose utilization in vivo and found that glucose metabolism in $\mathrm{T}$ cells changes dynamically over an immune response ${ }^{43}$. Early effectors undergo clonal expansion and demand glucose for bioenergy and biosynthesis ${ }^{43}$, which causes glucose limitation at effector sites. Late effector $\mathrm{T}$ cells show lower biosynthetic glucose metabolism than early effectors do because of lower proliferation rates or glucose limitation ${ }^{43}$. Production of the glycolytic metabolite FBP may change along with the course of immune response due to the glucose metabolic alterations. Early effector T cells possess increased glycolysis and FBP production, which suppresses AMPK coupled SENP1-Sirt3 signalling at this status. At the late phase, glycolysis is markedly reduced in effector T cells, resulted in downregulated FBP production, which releases the inhibition from AMPK coupled SENP1-Sirt3 signalling for memory development. We thus reveal a critical role of FBP as a metabolite in controlling $\mathrm{T}$ cell fate during immune response.

AMPK is a crucial regulator in effector $\mathrm{T}$ cell metabolic adaptation to nutrient starvation ${ }^{22}$. AMPK signalling in effector T cells downregulates mTORC1 activity, which in turn diminishes energy expenditure and activates glutamine-dependent OXPHOS instead of glucose for ATP production ${ }^{22}$. Compared with that in effector cells, AMPK activation is increased in memory $\mathrm{T}$ cells ${ }^{14,23,54}$. We demonstrated that glucose limitation induced AMPK activation, which activated SENP1-Sirt3 signalling for $\mathrm{T}$ cell memory development at late phase of effector immune response. SENP1-Sirt3 signalling rescued AMPK inhibition-diminished memory development, suggesting that SENP1-Sirt3 signalling is a crucial downstream event following AMPK activation in memory development.

We revealed that the SENP1-Sirt3 axis activated OXPHOS and mitochondrial fusion in $\mathrm{T}$ cells, and this activation increased $\mathrm{T}$ cell survival and memory development. Buck et al. reported that OPA1-mediated mitochondrial fusion was required for $\mathrm{T}_{M}$ cell development ${ }^{7}$. YME1L1 and OMA1 are two proteases that cleave L-OPA1 to generate S-OPA1 ${ }^{48,49}$. In this study, we identified YME1L1 but not OMA1 as a critical substrate of Sirt3 for deacetylation to promote mitochondrial fusion. YME1L1 acetylation mutant represented inhibited SENP1-Sirt3 axis in modulating mitochondrial fusion and memory $\mathrm{T}$ cell survival, suggesting that deacetylation of YME1L1 by SENP1-Sirt3 signalling is a critical regulation mechanism in controlling OPA1 cleavage and subsequent OPA1-mediated mitochondrial fusion in T cell memory development. Meanwhile, the increased cristae in SENP1-Sirt3mediated mitochondrial fusion provide a structural basis for high OXPHOS activity shown in memory T cells. Therefore, YME1L1 may play a crucial role in OPA1-mediated mitochondrial fusion and $\mathrm{T}$ cell memory development.

Our findings may contribute to rescuing $\mathrm{T}$ cell dysfunction in the tumour microenvironment. In solid tumours, tumour cells consume many more nutrients to meet their proliferation demands, which results in the depletion of glucose and other nutrients in the tumour microenvironment ${ }^{17,18}$. This unique feature of the tumour microenvironment can suppress $\mathrm{T}$ cell activity $^{17}$ and also limits its anti-tumour immune efficacy ${ }^{18}$. In this study, we found that SENP1-Sirt3 signalling promoted T cell infiltration into tumour tissue and enhanced anti-tumour immunity. We also showed that metformin activated AMPKSENP1-Sirt3 signalling in T cells. Therefore, we hypothesize that the activation of AMPK-SENP1-Sirt3 signalling in $\mathrm{T}$ cells is a potent strategy for anti-tumour therapy. It is expected that this strategy combined with anti-PD-1/PD-L1 immunotherapy may induce the anti-tumour immunity of $\mathrm{T}$ cells.

\footnotetext{
Methods

Mice. C57BL/6 Sirt3 wild-type and K223R mice were described in our previous studies $^{31}$. C57BL/6 CD45.1 mice (Jackson Laboratory), Cd4-Cre mice (Jackson Laboratory) and major histocompatibility complex (MHC) class I-restricted OVAspecific TCR OT1 transgenic mice (Jackson Laboratory) were obtained from Shanghai Institute of Immunology ${ }^{11}$. C57BL/6 Sirt3-knockout (Sirt3 KO) mice were obtained from Prof. Shimin Zhao's Laboratory (Fudan University, China) ${ }^{55}$. Sirt3 WT or K223R mice were crossed to C57BL/6 OT1 transgenic mice to generate Sirt3 WT or K223R OT1 mice. To create T cell-specific Senp1 cKO mice (Senp1 flox) flox $\times$ Cd4-Cre), Senp1 flox/flox mice were crossed with Cd4-Cre mice. Senp1 flox/flox
} 
a
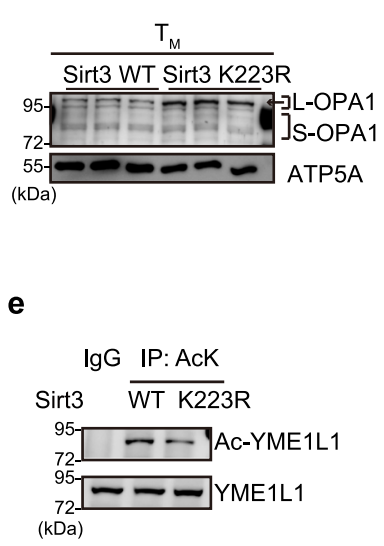

b

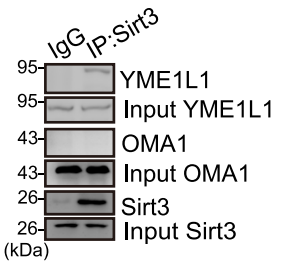

f

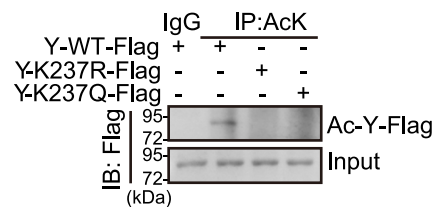

C

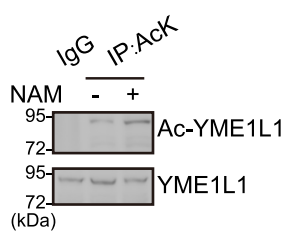

d

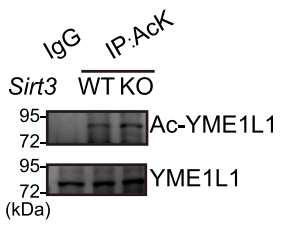

$\mathbf{g}$

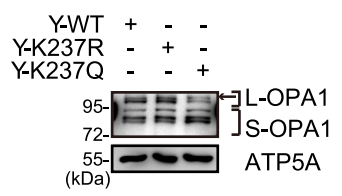

h

Y-WT

Y-K237R
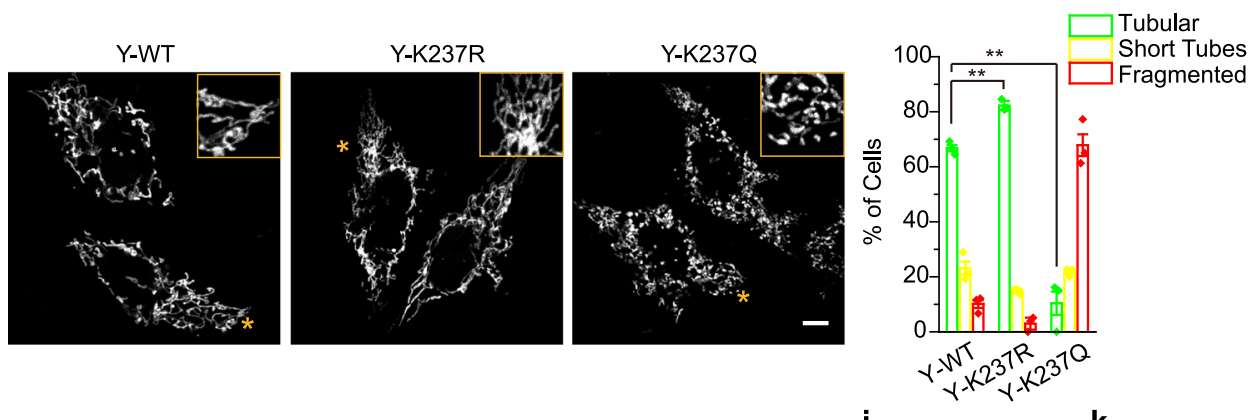

i
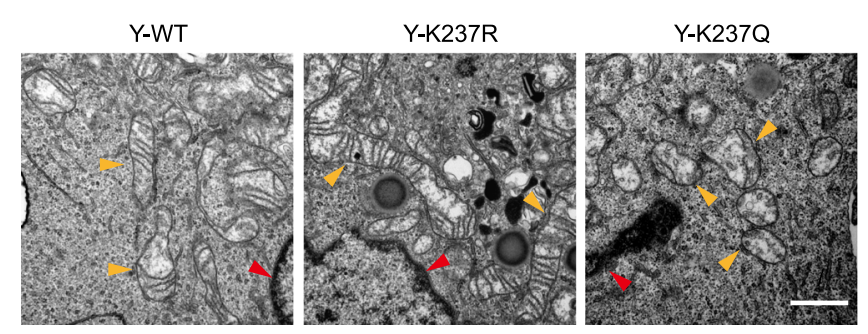

j

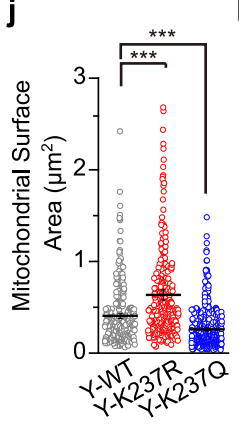

k

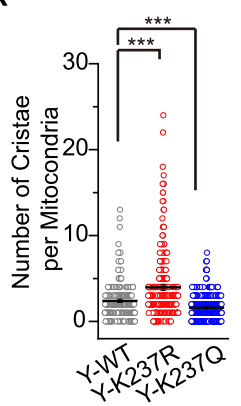

I

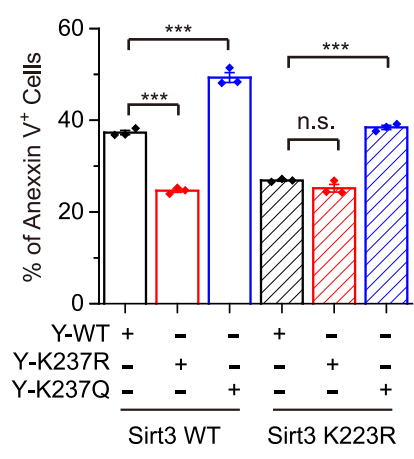

m

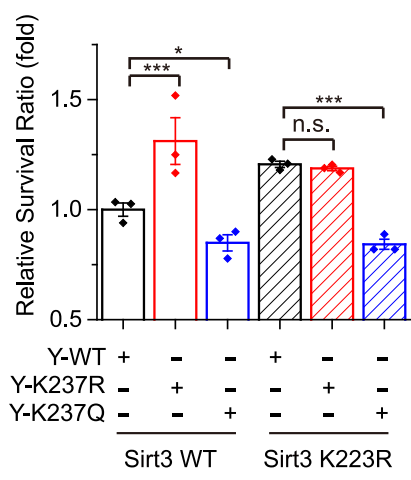

mice produced in previous studies ${ }^{56,57}$. All mice were bred and maintained under specific pathogen free (SPF) conditions. All mice were bred and maintained under a $12 \mathrm{~h}$ reverse light/dark cycle with a temperature of $22 \pm 2{ }^{\circ} \mathrm{C}$ and a humidity of $40-70 \%$. Age and sex matched male and female adult (6-8 week old) mice were used in each independent experiment. The animal experiments were performed in strict accordance with the "Guide for the Care and Use of Laboratory Animals", which were approved by the Experimental Animal Ethical Committee at Shanghai Jiao Tong University School of Medicine.

Cell culture. HEK-293T (ATCC CRL-11268), HeLa (ATCC CCL-2) and OVAexpressing mouse MC38 (MC38-OVA) cells were cultured in Dulbecco's modified Eagle's medium (DMEM, $4500 \mathrm{mg} / \mathrm{l}$ glucose, $4 \mathrm{mM}$ L-glutamine). Jurkat Clone E61 (ATCC TIB-152) cells were cultured in RPMI 1640, containing $10 \%$ fetal bovine serum (FBS), 100 unit/ml penicillin and $100 \mu \mathrm{g} / \mathrm{ml}$ streptomycin. MC38-OVA cells were made by Yang-Xin Fu Lab (UT South western Medical Center, USA) ${ }^{58}$, and were obtained from Shanghai Institute of Immunology. All of the cell lines are mycoplasma free. YME1L1-knocked out HeLa cells were generated by CRISPRCas9 technology with a specific gRNA (ggaaccgaccatattacaacagg).

Plasmids. We used primers 5'-atatgcggccgcatgttttccttgtcgagcac-3' (sense) and 5'atatgcggccgctatatctcacttccaac-3' (anti-sense) to amplify YME1L1 from Human cDNA, and constructed YME1L1-Flag in pcDNA3.1 (+) and pCDH-GFP using standard PCR-based cloning strategies. We mutated YME1L1 K237 to R with primers 5'-ccatcattcgtgagggggtttcttttg-3' (sense) and 5'-caaacgaaacccctcacgaatgatgg-3' (anti-sense), and mutated K237 to Q with primers 5'-ccatcattcgtgcaggggtttcttttg-3' (sense) and 5'-caaacgaaaccctgcacgaatgatgg-3' (anti-sense). All 
Fig. 5 Sirt3 regulates YME1L1-mediated OPA1 processing and mitochondrial fusion. a Mitochondrial lysates from Sirt3 WT or K223R $T_{M}$ cells were blotted with anti-OPA1 antibody ( $n=3$ biologically independent samples). b Mitochondrial lysates from Jurkat cells were immunoprecipitated with antiSirt3 antibody and blotted with anti-YME1L1 and -OMA1 antibodies. c-f YME1L1 acetylation level in NAM (5 mM, 8 h) treated Jurkat cells (c), Sirt3 WT or KO TM cells (d), Sirt3 WT or K223R $T_{M}$ cells (e), in Flag-YME1L1 WT (Y-WT), YME1L1 K237R (Y-K237R) or YME1L1 K237Q (Y-K237Q) plasmids transfected 293 T cells (f). $\mathbf{g}$ OPA1 protein in HeLa cells infected with Y-WT, Y-K237R or Y-K237Q lentivirus. $\mathbf{h}$ The percentages of HeLa cells with tubular, short-tube-shaped or fragmented mitochondria were determined with MitoTracker staining $\left(n=3\right.$ independent experiment, ${ }^{\star \star} P=0.0012, Y-W T$ vs $Y$ $\mathrm{K} 237 \mathrm{R} ;{ }^{\star \star} P=0.0056, \mathrm{Y}-\mathrm{WT}$ vs $\mathrm{Y}-\mathrm{K} 237 \mathrm{Q}$ for tubular). The area denoted by the asterisk is enlarged on the top right, and the scale bars, $10 \mu \mathrm{m}$. $\mathbf{i}-\mathbf{k}$ The mitochondrial microscopy of Jurkat cells infected with Y-WT, Y-K237R or Y-K237Q lentivirus (i). The mitochondrial surface area ( $\mathbf{j}$ ) and the number of

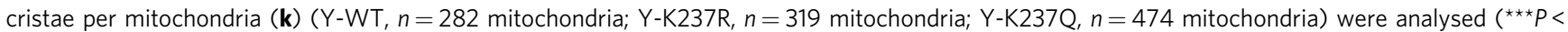
$0.0001, Y-W T$ vs $Y-K 237 R ;{ }^{\star \star \star} P<0.0001, Y-W T$ vs $Y-K 237 Q(\mathbf{j}) ;{ }^{\star \star \star} P<0.0001, Y-W T$ vs $Y-K 237 R ;{ }^{\star \star \star} P<0.0001, Y-W T$ vs $Y-K 237 Q(\mathbf{k})$ ). The scale bars, $1 \mu \mathrm{m}$. I, $\mathbf{m}$ Sirt3 WT or K223R $T_{M}$ cells were infected with lentivirus (Lv) containing Y-WT, Y-K237R or Y-K237Q ( $n=3$ biologically independent samples). The apoptotic ratio ( ${ }^{\star \star \star} P<0.0001, Y-W T$ vs $Y-K 237 R ;{ }^{* \star \star} P<0.0001, Y-W T$ vs $Y-K 237 Q$ in Sirt3 WT; ${ }^{* \star \star} P<0.0001, Y-W T$ vs $Y-K 237 Q$ in Sirt3 $\mathrm{K} 223 \mathrm{R})(\mathrm{I})$, and the survival ratio ( ${ }^{\star \star \star} P=0.0007, \mathrm{Y}-\mathrm{WT}$ vs $\mathrm{Y}-\mathrm{K} 237 \mathrm{R} ;{ }^{\star} P=0.0499, \mathrm{Y}-\mathrm{WT}$ vs $\mathrm{Y}-\mathrm{K} 237 \mathrm{Q}$ in Sirt3 WT; ${ }^{\star \star \star} P=0.0002, \mathrm{Y}-\mathrm{WT}$ vs $\mathrm{Y}-\mathrm{K} 237 \mathrm{Q}$ in Sirt3 K223R) (m) were measured. All immunoblots are representative of three independent experiments (a-g). Two-way ANOVA followed by Fisher's LSD test $(\mathbf{h}, \mathbf{j}-\mathbf{m})$. All data are shown as the means \pm SEMs. n.s. not significant.

plasmids were verified by DNA sequencing. We used pCDH lentivirus system (System Biosciences, CD511B-1) to produce lentiviral-GFP-YME1L1, -YME1L1 K237R, or -YME1L1 K237Q virus according to the manufacture manual.

T cell culture and activation. CD8 + OT1 naive $\mathrm{T}$ cells were isolated from splenocytes. For $\mathrm{T}_{\mathrm{E}}$ or $\mathrm{T}_{\mathrm{M}}$ cell development, these naive $\mathrm{T}$ cells were activated with $\mathrm{OVA}_{257-264}$ peptide (SIINFEKL, Sigma) or anti-CD3 $(5 \mu \mathrm{g} / \mathrm{ml}) / \mathrm{anti}-\mathrm{CD} 28(2 \mu \mathrm{g} /$ $\mathrm{ml})$, with IL-2 (100 U/ml, PeproTech) for 3 days and then cultured in the presence of either IL-2 $\left(100 \mathrm{U} / \mathrm{ml}\right.$, for $\left.\mathrm{T}_{\mathrm{E}}\right)$ or IL-15 $\left(10 \mathrm{ng} / \mathrm{ml}\right.$, for $\left.\mathrm{T}_{\mathrm{M}}\right)$ for another 3 days in $\mathrm{T}$ cell media (RPMI $1640+10 \% \mathrm{FBS}+100 \mathrm{U} / \mathrm{ml}$ penicillin/streptomycin $+2 \mathrm{mM}$ $\mathrm{L}$-glutamine $+55 \mu \mathrm{M} \beta$-mercaptoethanol) $)^{7,13-15}$. For in vitro $\mathrm{T}_{\mathrm{M}}$ cell survival assays $7,13-15, \mathrm{~T}_{\mathrm{M}}$ cell was cultured in 96 -well $\left(1 \times 10^{5}\right.$ cells/well $)$ round bottom plates in T cell medium without IL-15 for 3 days, respectively ${ }^{15}$. Using 7-AAD staining analyzed the survival ratio ${ }^{15}$. For differentiation of mouse Th1, Th2, Th17 and Treg cells from $\mathrm{CD} 4{ }^{+}$naïve T cells in vitro $(n=3$ biologically independent samples), purified $\mathrm{CD} 4{ }^{+}$naïve $\mathrm{T}$ cells (by EasySep Mouse Naive CD4 ${ }^{+} \mathrm{T}$ Cell Isolation Kit, STEMCELL, Cat\#19765) were stimulated by anti-CD3/CD28 + IL-2 and cultured in the presence of polarizing cytokines for 6 days: IL-12 $(20 \mathrm{ng} / \mathrm{mL}$, PeproTech, Cat\#210-12) + anti-IL-4 (10 ng/mL, Biolegend, Cat\#504101), for Th1 cells; or IL-4 (100 ng/mL, PeproTech, Cat\#214-14) + anti-IFN- $\gamma(10 \mathrm{ng} / \mathrm{mL}$, Biolegend, Cat\#517903) and anti-IL-12(10 ng/mL, Biolegend, Cat\#505303), for Th2 cells; or TGF- $\beta 1$ (5 ng/mL, R\&D System, Cat\#7666-MB-005) + anti-IL-4 \& antiIFN- $\gamma(10 \mathrm{ng} / \mathrm{mL})$, for Treg cells; or TGF- $\beta 1(1 \mathrm{ng} / \mathrm{mL})+\mathrm{IL}-6(100 \mathrm{ng} / \mathrm{mL}$, PeproTech, Cat\#216-16) + anti-IL-4 \& anti-IFN- $\gamma(10 \mathrm{ng} / \mathrm{mL})$, for Th17 cells.

T cell adoptive transfer. For in vivo $\mathrm{T}_{\mathrm{M}}$ cell survival assays $\mathrm{s}^{7,13-15}$, OT1 CD8 ${ }^{+} \mathrm{T}_{\mathrm{M}}$ cells from donor splenocytes (CD45.2) were isolated and transferred intravenously (i.v.) into congenic recipient mice (C57BL/6, CD45.1). For anti-tumor immunity assays, the $\mathrm{CD} 8{ }^{+} \mathrm{T}_{\mathrm{M}}$ cells or $\mathrm{OVA}_{257-264}$ activated OT1 $\mathrm{T}$ cells $\left(\mathrm{H}-2 \mathrm{~Kb}^{+}, \mathrm{C} 57 \mathrm{BL} / 6\right)$ were transferred intravenously (i.v.) into congenic recipient mice (C57BL/6) ${ }^{7}$.

LM-OVA infection. Listeria monocytogenes expressing-OVA (LM-OVA) was obtained from Dr. Shen's laboratory ${ }^{41}$, and cultured in Brain-Heart Infusion(BHI) medium with $50 \mu \mathrm{g} / \mathrm{ml}$ Streptomycin. $\mathrm{CD}^{+}$naïve T cells $\left(1.0 \times 10^{4}\right)$ from Sirt3 WT-OT1 and Sirt3 K223R OT1 were adoptive transferred into congenic recipient mice (C57BL/6). The recipient mice then were either injected (i.v.) with a sublethal dose of LM-OVA $\left(1.0 \times 10^{5} \mathrm{CFU}\right)^{14}$.

T cell lentiviral transduction. Briefly, $\mathrm{CD} 8+\mathrm{T}_{\mathrm{M}}$ cells generated from naive $\mathrm{T}$ cells using in vitro standard procedure were infected with the packaged lentivirus in the presence of $10 \mathrm{mg} / \mathrm{ml}$ polybrene by spinning at 1200 r.p.m. for $90 \mathrm{~min}$ at $30^{\circ} \mathrm{C}^{59}$. Supernatant was removed after infection and replaced with $\mathrm{T}$ cell medium containing IL-15 $(10 \mathrm{ng} / \mathrm{ml})$ for $48 \mathrm{~h}$ (infection efficient $>80 \%)^{59}$. Then we collected the infected $\mathrm{T}_{\mathrm{M}}$ cells for apoptosis staining and 7-AAD based in vitro survival experiments.

Mitochondria isolation. The procedure used for mitochondria isolation was described in our previous study ${ }^{31}$. Briefly, cells were incubated in ice-cold isolation buffer TD [135 mM NaCl, $5 \mathrm{mM} \mathrm{KCl}, 25 \mathrm{mM}$ Tris- $\mathrm{HCl}$ (pH 7.5)] containing complete EDTA-free protease inhibitor mixture (Roche). After centrifuging at $600 \times g$ for $10 \mathrm{~min}$, the pellet was washed by $10 \mathrm{ml}$ ice-cold TD buffer and centrifuged again at $600 \times g$ for $10 \mathrm{~min}$. The pellet was then re-suspended in ice-cold isolation buffer MS containing $210 \mathrm{mM}$ mannitol, $70 \mathrm{mM}$ sucrose, $5 \mathrm{mM}$ Tris- $\mathrm{HCl}$ (pH 7.5), $1 \mathrm{mM}$ EGTA, $0.5 \mathrm{mg} / \mathrm{ml}$ BSA and protease inhibitor mixture. The lysate was further homogenized in this buffer with a Glass-Teflon motorized homogenizer for 40 times. The mitochondrial fraction was isolated by differential centrifugation $1300 \times g$ for $5 \mathrm{~min}$ and $17,000 \times g$ for $15 \mathrm{~min}$. Subsequently, the pellet was re-suspended in $1.5 \mathrm{M}$ sucrose solution containing $1 \mathrm{mM}$ EGTA and $10 \mathrm{mM}$ Tris- $\mathrm{HCl}(\mathrm{pH} 7.5)$. A thin layer was formed after gently adding $1 \mathrm{M}$ sucrose solution on the suspension solution. This layer was drawn out from the tube and diluted by 4 volume dilution buffers [ $1 \mathrm{mM}$ EGTA and $5 \mathrm{mM}$ Tris- $\mathrm{HCl}$ ( $\mathrm{pH}$ 7.5)] to the final concentration of $250 \mathrm{mM}$ sucrose. After centrifugation at $17,000 \times g$ for $15 \mathrm{~min}$, the purified mitochondria were dissolved in MS buffer for $-80^{\circ} \mathrm{C}$ stock.

Metabolomics. The procedure used for mitochondria metabolomics analysis is described elsewhere ${ }^{31}$. Mitochondrial lysates were precipitated with acetonitrile containing internal standards; the supernatant was separated by centrifugation, and then analyzed by tandem mass spectrometry (TSQ Vantage, Thermo Fisher Scientific). Chromatographic separation was performed on a XBridgeTM Amide column $(2.1 \times 150 \mathrm{~mm}, 5 \mu \mathrm{m}$; Waters $)$ with a XBridgeTM Amide guard column $(2.1 \times 10 \mathrm{~mm}, 5 \mu \mathrm{m}$; Waters). The mobile phase was composed of $10 \mathrm{mM}$ ammonium acetate in water (phase A) and acetonitrile (phase B). L-carnitine, acylcarnitines and their internal standards (Sigma-Aldrich) were analyzed in positive ion multiple reaction monitoring (MRM) mode. The raw data files were processed using LCquan 2.7 software (Thermofisher Scientific) to generate chromatographic peak areas of each analyst and their response ratios to internal standards. Then the concentration of $\mathrm{C} 0$ and acylcarnitines were calculated from the response ratios and the concentration of their internal standards using isotope dilution method.

Detection of fructose-1,6-bisphosphate, ATP and acetyl-CoA. Concentration of ATP in T cell mitochondria was determined using an ATP Colorimetric/Fluorometric Assay Kit (Cat\#K354-100, Biovision). Concentration of Acetyl-CoA in T cell mitochondria was determined using a PicoProbe ${ }^{\mathrm{TM}}$ Acetyl-CoA Fluorometric assay kit (Cat\#K317-100, BioVision). Concentration of Fructose-1,6-Bisphosphate (FBP) in pretreated T cells was determined using a PicoProbe Fructose-1,6Bisphosphate Assay Kit (Cat\#K2036, Biovision).

Flow cytometry. Fluorochrome-labelled antibodies (Biolegend, eBioscience and BD Pharmingen), MitoTracker (Green/Red/Deep Red), or JC-1 (Invitrogen) staining were performed according to the manufacturer's instructions. OVAspecific $\mathrm{CD}^{+}{ }^{+} \mathrm{T}$ cells from blood or MC38-OVA tumor were staining with $\mathrm{H} 2-\mathrm{Kb}$ $\mathrm{OVA}_{\text {257-264 }}$ MHC-peptide tetramers (MBL, Japan). Cells were collected on BD FACSVerse ${ }^{\mathrm{TM}}$ with BD FACSuite (1.0.5.3841) software or LSR Fortessa X-20 cell analyzer (BD Biosciences) and BD FACSDiva (6.1.3) software, and analyzed using FlowJo (TreeStar) software. $\mathrm{CD} 8^{+}$naive T Cells $\left(\mathrm{CD} 8^{+} \mathrm{CD} 44^{-} \mathrm{CD} 62 \mathrm{~L}^{+}\right)$were sorted using a MoFlo Astrios (BeckMan) or isolated by EasySep Mouse Naive CD8 $+\mathrm{T}$ Cell Isolation Kit (STEMCELL).

Imaging. For mitochondria observation by confocal fluorescence microscopy, $\mathrm{T}_{M}$ cells or HeLa cells were stained with MitoTracker Red (Invitrogen) at 1:2000 dilution for $30 \mathrm{~min}$, followed by the fixation before nuclear counterstaining with DAPI or Hoechst. Images were acquired using a Leica TCS Sp8 STED confocal microscope, and a Leica LAS X Core (3.7.2.22383) software. For transmission electron microscopy, $\mathrm{T}_{\mathrm{M}}$ or Jurkat cells were fixed in $2.5 \%$ glutaraldehyde. Cut sections were imaged using a HITACHI H-7650 or PHILIPS CM-120 transmission electron microscope ${ }^{31}$. RADIUS 2.0 software (EMSIS GmbH, Muenster, Germany) software was used for transmission electron microscope data collection. Image-J (1.52a), Image Pro-Plus (version 6.0), Adobe Photoshop CS6 and Adobe Illustrator CS6 were used to analyse and process fluorescence and electron microscopy images.

Seahorse analysis. Oxygen consumption rates (OCR) and extracellular acidification rates (ECAR) were measured using a 96-well XF Extracellular Flux 
a

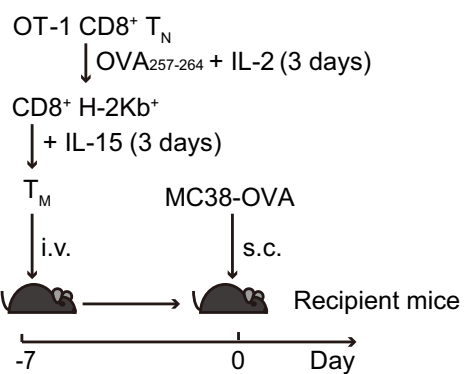

d

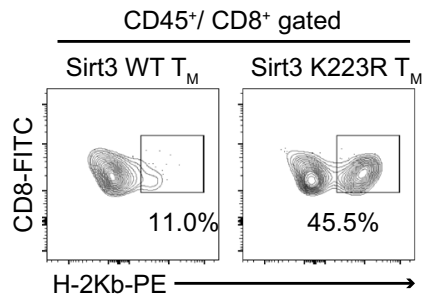

f

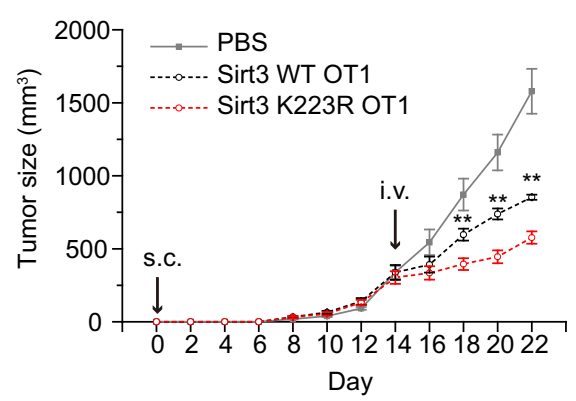

i b

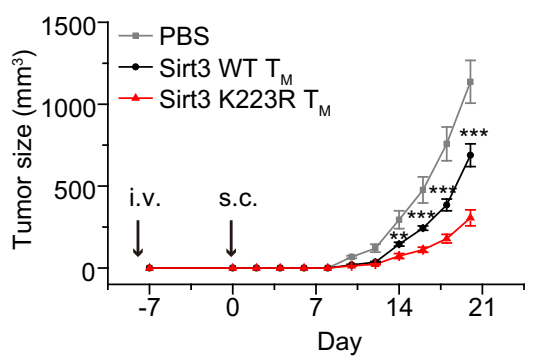

C

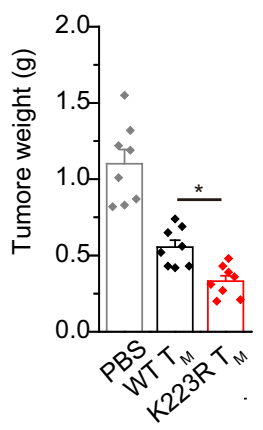

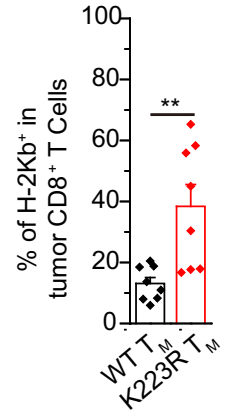

g

e

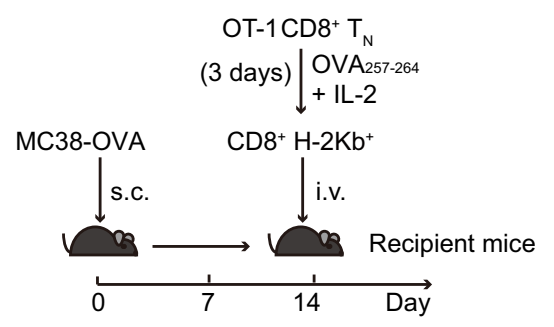

h

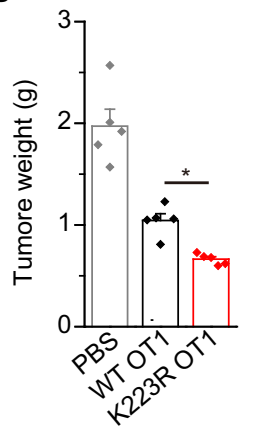

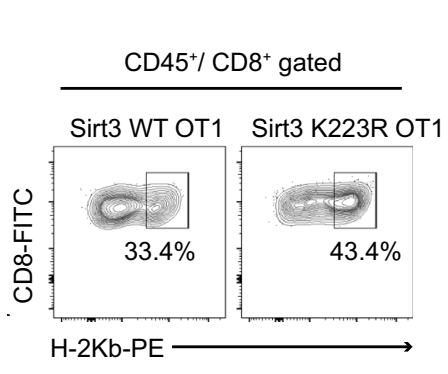
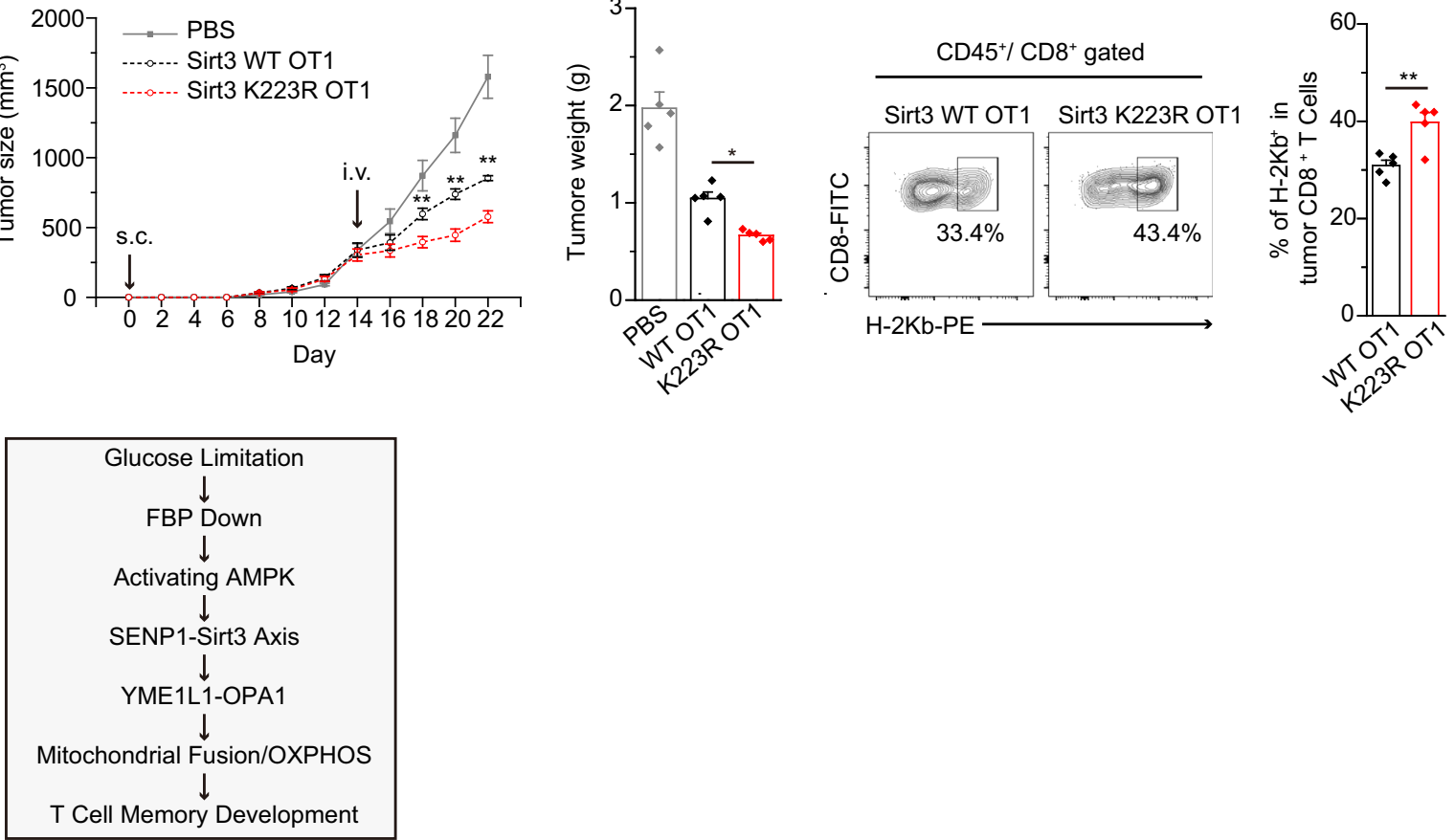

Fig. 6 Sirt3 K223R enhances the anti-tumour immunity of CD8 ${ }^{+} \mathbf{T}$ cells. a-d Sirt3 WT or K223R OT1 T $M$ cells $\left(1.5 \times 10^{6}\right)$ were transplanted into congenic recipient mice (C57BL/6). Seven days' post-transplantation, MC38-OVA tumour cells $\left(1.0 \times 10^{5}\right)$ were subcutaneously inoculated into the recipient mice ( $n=8$ mice per group) (a). The tumour growth curve is shown in (Two-way ANOVA followed by Fisher's LSD test: ${ }^{\star \star} P=0.0026,{ }^{\star \star \star} P<0.0001,{ }^{\star \star \star} P=$ 0.0005 and ${ }^{\star \star \star} P=0.0007$ on day 14 , day 16 , day 18 and day 20, respectively) (b). The tumour weight was measured at day 20 post-injection (One-way ANOVA followed by Fisher's LSD test: ${ }^{\star} P=0.021$ for Sirt3 WT $T_{M}$ vs Sirt3 K223R WT T $T_{M}$ ) (c). The frequency of donor $\mathrm{CD}^{+}{ }^{+} \mathrm{T}$ cells $\left(\mathrm{H}-2 \mathrm{~Kb} \mathrm{~b}^{+}\right)$in the tumours of the recipient mice was assessed by FACS (Two-tailed unpaired $t$-test: $\left.{ }^{\star \star} P=0.0041\right)(\mathbf{d})$. e-h MC38-OVA tumour cells $\left(1.0 \times 10^{5}\right)$ were planted subcutaneously into recipient mice (C57BL/6). OVA $254-264$ activated OT1-T cells $\left(1 \times 10^{7}\right)$ were freshly obtained from Sirt3 WT or K223R OT1 naïve T cells and intravenously (i.v.) transplanted into the above-mentioned mice at day 14 after the injection of tumour cells ( $n=5$ mice per group) (e). The tumour growth curve was recorded (Two-way ANOVA followed by Fisher's LSD test: ${ }^{\star \star} P=0.008,{ }^{\star \star} P=0.0011$ and ${ }^{\star \star} P=0.0013$ on day 18 , day 20 and day 22 , respectively) $(\mathbf{f})$. The tumour volume $\left(\mathrm{mm}^{3}\right)$ was calculated using the following equation: (length $\times$ width $\left.{ }^{2}\right) / 2$. The tumour weight was measured at day 22 after the injection of tumour cells (One-way ANOVA followed by Fisher's LSD test: ${ }^{\star} P=0.025$ for Sirt3 WT-OT1 vs Sirt3 K223R OT1) (g). The frequency of donor $\mathrm{CD} 8^{+} \mathrm{T}$ cells $\left(\mathrm{H}-2 \mathrm{~Kb}^{+}\right)$isolated from the tumours in the recipient mice was determined by FACS analysis (Two-tailed unpaired $t$-test: ${ }^{\star \star} P=$ 0.0047) (h). i Model depicting the role of the AMPK-SENP1-Sirt3 axis in regulating FAO-fuelled OXPHOS and mitochondrial fusion in CD8 ${ }^{+} \mathrm{T}_{M}$ cells. 
Analyzer (EFA) (Seahorse Bioscience) as described as previous study, and Seahorse Wave (2.6.1.53) software was used for data collection ${ }^{7,15,31}$. XF media contains non-buffered RPMI 1640, $25 \mathrm{mM}$ glucose (for OCR), $2 \mathrm{mM} \mathrm{L}$-glutamine and $1 \mathrm{mM}$ sodium pyruvate. One micromolar oligomycin, $1.5 \mu \mathrm{M}$ fluoro-carbonyl cyanide phenylhydrazone (FCCP), or $100 \mathrm{nM}$ rotenone $+1 \mu \mathrm{M}$ antimycin A were used in these assays according to the manufacturer's instructions.

Statistics and reproducibility. All experiments were performed with at least three biological replicates as indicated in the figure legends. All co-immunoprecipitation and immunoblots were repeated at least three times independently with similar results. Comparisons for two groups were calculated using paired or unpaired twotailed student's $t$-tests, as appropriate. And comparisons for more than two groups were calculated using one-way or two-way ANOVA followed by Fisher's LSD multiple comparison tests, as appropriate. Statistical analysis was performed using GraphPad Prism. The Details of statistical testing and the exact $P$-value are provided in figure legends. The level of significance is indicated as follows: ${ }^{*} P<0.05$, $* * P<0.01, * * * P \leq 0.001$.

Reporting summary. Further information on research design is available in the Nature Research Reporting Summary linked to this article.

\section{Data availability}

All data are provided in the article and Supplementary files or are available from the corresponding author on reasonable request. Source data are provided with this paper.

Received: 24 October 2020; Accepted: 22 June 2021;

Published online: 16 July 2021

\section{References}

1. Joshi, N. S. et al. Inflammation directs memory precursor and short-lived effector CD8(+) T cell fates via the graded expression of T-bet transcription factor. Immunity 27, 281-295 (2007).

2. Wei, J. et al. Targeting REGNASE-1 programs long-lived effector T cells for cancer therapy. Nature 576, 471-476 (2019).

3. Reading, J. L. et al. The function and dysfunction of memory CD8+ T cells in tumor immunity. Immunol. Rev. 283, 194-212 (2018).

4. Sukumar, M. et al. Inhibiting glycolytic metabolism enhances CD8+ T cell memory and antitumor function. J. Clin. Invest. 123, 4479-4488 (2013).

5. Pan, Y. et al. Survival of tissue-resident memory T cells requires exogenous lipid uptake and metabolism. Nature 543, 252-256 (2017).

6. Phan, A. T. et al. Constitutive glycolytic metabolism supports CD8+ T cell effector memory differentiation during viral infection. Immunity $\mathbf{4 5}$, 1024-1037 (2016).

7. Buck, M. D. et al. Mitochondrial dynamics controls T cell fate through metabolic programming. Cell 166, 63-76 (2016).

8. Pollizzi, K. N. et al. Asymmetric inheritance of mTORC1 kinase activity during division dictates CD8(+) T cell differentiation. Nat. Immunol. 17, 704-711 (2016).

9. Chang, C. H. et al. Posttranscriptional control of T cell effector function by aerobic glycolysis. Cell 153, 1239-1251 (2013).

10. Gubser, P. M. et al. Rapid effector function of memory CD8+ T cells requires an immediate-early glycolytic switch. Nat. Immunol. 14, 1064-1072 (2013).

11. $\mathrm{Hu}, \mathrm{Z}$. et al. Acylglycerol kinase maintains metabolic state and immune responses of CD8(+) T cells. Cell Metab. 30, 290-302 (2019).

12. Lochner, M., Berod, L. \& Sparwasser, T. Fatty acid metabolism in the regulation of T cell function. Trends Immunol. 36, 81-91 (2015).

13. O'Sullivan, D. et al. Memory CD8+ T cells use cell-intrinsic lipolysis to support the metabolic programming necessary for development. Immunity 41, 75-88 (2014)

14. Pearce, E. L. et al. Enhancing CD8 T-cell memory by modulating fatty acid metabolism. Nature 460, 103-107 (2009).

15. van der Windt, G. J. et al. Mitochondrial respiratory capacity is a critical regulator of CD8+ T cell memory development. Immunity 36, 68-78 (2012).

16. Pearce, E. L., Poffenberger, M. C., Chang, C. H. \& Jones, R. G. Fueling immunity: insights into metabolism and lymphocyte function. Science 342, 1242454 (2013).

17. Scharping, N. E. et al. The tumor microenvironment represses $\mathrm{T}$ cell mitochondrial biogenesis to drive intratumoral $\mathrm{T}$ cell metabolic insufficiency and dysfunction. Immunity 45, 374-388 (2016).

18. Zhang, Y. et al. Enhancing $\operatorname{CD} 8(+) \mathrm{T}$ cell fatty acid catabolism within a metabolically challenging tumor microenvironment increases the efficacy of melanoma immunotherapy. Cancer Cell 32, 377-391 (2017).

19. Wai, T. \& Langer, T. Mitochondrial dynamics and metabolic regulation. Trends Endocrinol. Metab. 27, 105-117 (2016).
20. Cao, Y. L. et al. MFN1 structures reveal nucleotide-triggered dimerization critical for mitochondrial fusion. Nature 542, 372-376 (2017).

21. Ban, T. et al. Molecular basis of selective mitochondrial fusion by heterotypic action between OPA1 and cardiolipin. Nat. Cell Biol. 19, 856-863 (2017).

22. Blagih, J. et al. The energy sensor AMPK regulates T cell metabolic adaptation and effector responses in vivo. Immunity 42, 41-54 (2015).

23. Araki, K. \& Ahmed, R. AMPK: a metabolic switch for CD8+ T-cell memory. Eur. J. Immunol. 43, 878-881 (2013).

24. Carrico, C., Meyer, J. G., He, W., Gibson, B. W. \& Verdin, E. The mitochondrial acylome emerges: proteomics, regulation by sirtuins, and metabolic and disease implications. Cell Metab. 27, 497-512 (2018).

25. Liu, X. et al. Acetate production from glucose and coupling to mitochondrial metabolism in mammals. Cell 175, 502-513 (2018). e513.

26. Hebert, A. S. et al. Calorie restriction and SIRT3 trigger global reprogramming of the mitochondrial protein acetylome. Mol. Cell 49, 186-199 (2013).

27. Dittenhafer-Reed, K. E. et al. SIRT3 mediates multi-tissue coupling for metabolic fuel switching. Cell Metab. 21, 637-646 (2015).

28. Ahn, B. H. et al. A role for the mitochondrial deacetylase Sirt3 in regulating energy homeostasis. Proc. Natl Acad. Sci. USA 105, 14447-14452 (2008).

29. Hirschey, M. D. et al. SIRT3 regulates mitochondrial fatty-acid oxidation by reversible enzyme deacetylation. Nature 464, 121-125 (2010).

30. Hirschey, M. D., Shimazu, T., Capra, J. A., Pollard, K. S. \& Verdin, E. SIRT1 and SIRT3 deacetylate homologous substrates: AceCS1,2 and HMGCS1,2. Aging 3, 635-642 (2011).

31. Wang, T. et al. SENP1-Sirt3 signaling controls mitochondrial protein acetylation and metabolism. Mol. Cell 75, 823-834 (2019).

32. He, J., Cheng, J. \& Wang, T. SUMOylation-mediated response to mitochondrial stress. Int. J. Mol. Sci. 21, 5657 (2020).

33. Cheng, A. et al. Mitochondrial SIRT3 mediates adaptive responses of neurons to exercise and metabolic and excitatory challenges. Cell Metab. 23, 128-142 (2016).

34. Qiu, X., Brown, K., Hirschey, M. D., Verdin, E. \& Chen, D. Calorie restriction reduces oxidative stress by SIRT3-mediated SOD2 activation. Cell Metab. 12, 662-667 (2010).

35. Brown, K. D. et al. Activation of SIRT3 by the NAD $(+)$ precursor nicotinamide riboside protects from noise-induced hearing loss. Cell Metab. 20, 1059-1068 (2014).

36. Haigis, M. C., Deng, C. X., Finley, L. W., Kim, H. S. \& Gius, D. SIRT3 is a mitochondrial tumor suppressor: a scientific tale that connects aberrant cellular ROS, the Warburg effect, and carcinogenesis. Cancer Res. 72, 2468-2472 (2012).

37. Choudhary, C. et al. Lysine acetylation targets protein complexes and coregulates major cellular functions. Science 325, 834-840 (2009).

38. Wang, Q. et al. Acetylation of metabolic enzymes coordinates carbon source utilization and metabolic flux. Science 327, 1004-1007 (2010).

39. White, J. T., Cross, E. W. \& Kedl, R. M. Antigen-inexperienced memory CD8 $+\mathrm{T}$ cells: where they come from and why we need them. Nat. Rev. Immunol. 17, 391-400 (2017).

40. White, J. T. et al. Virtual memory T cells develop and mediate bystander protective immunity in an IL-15-dependent manner. Nat. Commun. 7, 11291 (2016).

41. Foulds, K. E. et al. Cutting edge: CD4 and CD8 T cells are intrinsically different in their proliferative responses. J. Immunol. 168, 1528-1532 (2002).

42. Zhang, C. S. et al. Fructose-1,6-bisphosphate and aldolase mediate glucose sensing by AMPK. Nature 548, 112-116 (2017).

43. Ma, E. H. et al. Metabolic profiling using stable isotope tracing reveals distinct patterns of glucose utilization by physiologically activated CD8(+) T cells. Immunity 51, 856-870 (2019).

44. Lazzarino, G. et al. Increase of intraerythrocytic fructose-1,6-diphosphate after incubation of whole human blood with fructose-1,6-diphosphate. Clin. Biochem. 17, 42-45 (1984).

45. Yakoub, K. M. et al. Fructose-1,6-bisphosphate protects hippocampal rat slices from NMDA excitotoxicity. Int. J. Mol. Sci. https://doi.org/10.3390/ ijms20092239 (2019).

46. Schluns, K. S. \& Lefrancois, L. Cytokine control of memory T-cell development and survival. Nat. Rev. Immunol. 3, 269-279 (2003).

47. Kurtulus, S., Tripathi, P. \& Hildeman, D. A. Protecting and rescuing the effectors: roles of differentiation and survival in the control of memory T cell development. Front. Immunol. 3, 404 (2012).

48. Anand, R. et al. The i-AAA protease YME1L and OMA1 cleave OPA1 to balance mitochondrial fusion and fission. J. Cell Biol. 204, 919-929 (2014).

49. Wai, T. et al. Imbalanced OPA1 processing and mitochondrial fragmentation cause heart failure in mice. Science 350, aad0116 (2015).

50. Civiletto, G. et al. Opal overexpression ameliorates the phenotype of two mitochondrial disease mouse models. Cell Metab. 21, 845-854 (2015).

51. Mishra, P., Carelli, V., Manfredi, G. \& Chan, D. C. Proteolytic cleavage of Opal stimulates mitochondrial inner membrane fusion and couples fusion to oxidative phosphorylation. Cell Metab. 19, 630-641 (2014). 
52. Ehses, $\mathrm{S}$. et al. Regulation of OPA1 processing and mitochondrial fusion by $\mathrm{m}$ AAA protease isoenzymes and OMA1. J. Cell Biol. 187, 1023-1036 (2009).

53. Yang, W. et al. Mitochondrial sirtuin network reveals dynamic SIRT3dependent deacetylation in response to membrane depolarization. Cell 167, 985-1000 (2016).

54. Rolf, J. et al. AMPKa1: a glucose sensor that controls CD8 T-cell memory. Eur. J. Immunol. 43, 889-896 (2013).

55. Hallows, W. C. et al. Sirt3 promotes the urea cycle and fatty acid oxidation during dietary restriction. Mol. Cell 41, 139-149 (2011).

56. Ferdaoussi, M. et al. Isocitrate-to-SENP1 signaling amplifies insulin secretion and rescues dysfunctional beta cells. J. Clin. Invest. 125, 3847-3860 (2015).

57. Wang, Y. et al. TRPV1 SUMOylation regulates nociceptive signaling in models of inflammatory pain. Nat. Commun. 9, 1529 (2018).

58. Mender, I. et al. Telomere stress potentiates STING-dependent anti-tumor immunity. Cancer Cell 38, 400-411 (2020). e406.

59. Rubinson, D. A. et al. A lentivirus-based system to functionally silence genes in primary mammalian cells, stem cells and transgenic mice by RNA interference. Nat. Genet. 33, 401-406 (2003).

\section{Acknowledgements}

This work was supported in part by the National Key Research and Development Program of China 2020YFA0803600 (J.C.), by National Natural Science Foundation of China 81730082 (J.C.), 82030075 (J.C.), 92049113 (T.W.), 32070759 (T.W.), 82003026 (J. H.), by Scientific Innovation Projects of Shanghai Education Committee 2017-01-07-0001-E00050 (J.C.), by Shanghai Municipal Education Commission, ZZjdyx15003 (T.W.), by Shanghai Jiao Tong University School of Medicine 18zxy004 (T.W.), YCTSQN2021005 (T.W.), by Shanghai Committee of Science and Technology 15ZR1424500 (T.W.), by Shanghai Jiaotong University Special Fund for Science and Technology Innovation Project 19X160010017 (J.C.) and by China Postdoctoral Science Foundation 2019M661548 (J.H.).

\section{Author contributions}

J.H., X.S., T.W., Q.W. and J.C. designed the research. J.H., X.S. and T.W. developed experimental methods, performed most experiments and analyse data. W.Z., Y.C., Q.Z., J.T., G.H., Z.L., C.J., L.D., S.W., Y.Z., R.C., Y.C., W.Y., B.D., W.X., H.T., Y.Q. and Q.F. provided technical supports. J.C. supervised the work. J.H., T.W. and J.C. wrote the manuscript. J.G., B.S., Y.E.C. and G.C. edited the manuscript.

\section{Competing interests}

The authors declare no competing interests.

\section{Additional information}

Supplementary information The online version contains supplementary material available at https://doi.org/10.1038/s41467-021-24619-2.

Correspondence and requests for materials should be addressed to Q.W., T.W. or J.C.

Peer review information Nature Communications thanks Ping-Chih Ho and the other, anonymous reviewer(s) for their contribution to the peer review of this work.

Reprints and permission information is available at http://www.nature.com/reprints

Publisher's note Springer Nature remains neutral with regard to jurisdictional claims in published maps and institutional affiliations.

(c) (i) Open Access This article is licensed under a Creative Commons Attribution 4.0 International License, which permits use, sharing, adaptation, distribution and reproduction in any medium or format, as long as you give appropriate credit to the original author(s) and the source, provide a link to the Creative Commons license, and indicate if changes were made. The images or other third party material in this article are included in the article's Creative Commons license, unless indicated otherwise in a credit line to the material. If material is not included in the article's Creative Commons license and your intended use is not permitted by statutory regulation or exceeds the permitted use, you will need to obtain permission directly from the copyright holder. To view a copy of this license, visit http://creativecommons.org/ licenses/by/4.0/.

(C) The Author(s) 2021 